This item was submitted to Loughborough's Research Repository by the author.

Items in Figshare are protected by copyright, with all rights reserved, unless otherwise indicated.

\title{
Underwater explosion of cylindrical charge near plates: Analysis of pressure characteristics and cavitation effects
}

PLEASE CITE THE PUBLISHED VERSION

https://doi.org/10.1016/j.ijimpeng.2018.06.009

\section{PUBLISHER}

(C) Elsevier

\section{VERSION}

AM (Accepted Manuscript)

\section{PUBLISHER STATEMENT}

This work is made available according to the conditions of the Creative Commons Attribution-NonCommercialNoDerivatives 4.0 International (CC BY-NC-ND 4.0) licence. Full details of this licence are available at: https://creativecommons.org/licenses/by-nc-nd/4.0/

\section{LICENCE}

CC BY-NC-ND 4.0

\section{REPOSITORY RECORD}

Zhang, Zhifan, Cheng Wang, L.K. Wang, A.M. Zhang, and Vadim Silberschmidt. 2019. "Underwater Explosion of Cylindrical Charge Near Plates: Analysis of Pressure Characteristics and Cavitation Effects". figshare. https://hdl.handle.net/2134/34668. 


\title{
Underwater explosion of cylindrical charge near plates: Analysis of pressure characteristics and cavitation effects
}

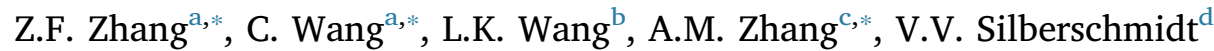 \\ a State Key Laboratory of Explosion Science and Technology, Beijing Institute of Technology, Beijing 100081, China \\ ${ }^{\mathrm{b}}$ China Ship Research and Development Academy, Beijing 100101, China \\ ${ }^{\mathrm{c}}$ College of Shipbuilding Engineering, Harbin Engineering University, Harbin 150001, China \\ ${ }^{\mathrm{d}}$ Wolfson School of Mechanical, Electrical and Manufacturing Engineering, Loughborough University, Leicestershire LE11 3TU, UK
}

A B S T R A C T

\begin{abstract}
In this paper, a coupled scheme utilizing advantages of the Runge-Kutta discontinuous Galerkin (RKDG) method and finite elements is applied to investigate cavitation induced by rarefaction waves during a near-field underwater explosion of cylindrical charge. A high-order RKDG method has advantages of an accurate shock capturing. So, it was used to solve a governing Eulerian equation for a compressible fluid. A finite-element method (FEM) was suitable to deal with problems of a shock response of structures and, therefore, applied for structural analysis. The suggested method was used to study pressure characteristics and cavitation effects of underwater explosions of cylindrical charges near single/double plates. First, a cavitation model was introduced in the RKDG method, and a numerical model of a high-pressure bubble in a cylinder was developed. The obtained numerical results were compared with the known solution in order to verify the validity of the suggested method. Second, a RKDG-FEM model of underwater explosion of a spherical charge near a plate was developed; its results for maximum deflection at the centre of the plate were compared with experimental data to prove the effectiveness of the coupled algorithm. Then, this algorithm was employed to simulate the process of underwater explosions of cylindrical charges near a single plate. Here, effects of different parameters - thickness of the plate and a distance between the charge and the plate - on pressure and cavitation characteristics were studied. Finally, a numerical model of double plates subjected to a near-field underwater explosion was developed. Cavitation evolution and its effect on shock-wave loading were analysed. Additionally, the effect of the distance between two plates was studied. The suggested analysis and its results provide a reference for load characteristics of near-field underwater explosions and shock response of structures.
\end{abstract}

Keywords:

RKDG-FEM

Cylindrical charge

Cavitation

Pressure characteristics

Shock response

\section{Introduction}

A high-pressure shock wave [1-5] can be generated in explosions caused by underwater weapons, such as torpedoes and bombs, causing severe damage to warships. Cylindrical charges are widely used in the design of such weapons, while they are usually substituted with spherical ones in research of underwater explosions. This is reasonable for a far-field underwater explosion; however, it is not suitable for a nearfield one. Cole [6] found that pressure evolution of a cylindrical charge was different from that of the equivalent spherical one when a distance to the explosive was less than 15 times a charge radius. A Lagrange method was used by Sternberg [7] to study the effect of a length-todiameter ratio of cylindrical charges on an underwater-explosion shock wave. Hammond [8] also investigated effects of parameters - charge shape and orientation for cylindrical geometry - on shock-wave characteristics. Huang et al. [9] experimentally studied bubble dynamics for cylindrical PETN with a length-to-diameter ratio from 3.35 to 6.75 subjected to underwater explosion. Zhang et al. [10] combined SPH and BEM to simulate the entire process of underwater explosion for a cylindrical charge and their numerical results agreed well with experimental data. So, it is of great significance to consider cylindrical characteristics of charges employed in near-field underwater explosions. Hence, a structural response of plates subjected to near-field underwater explosion of cylindrical charges is studied in this paper.

Rarefaction waves can be generated during the process of shockwave propagation between a bubble and a structure subjected to a nearfield underwater explosion, resulting in a cavitation region with low pressure [11-15]. As a result, the structure can be damaged by a secondary shock and reloading caused by collapse of this cavitation. Hence, it is necessary to investigate generation and collapse of

\footnotetext{
* Corresponding authors.

E-mail addresses: heuzhangzhifan@hotmail.com (Z.F. Zhang), wangcheng@bit.edu.cn (C. Wang), zhangaman@hrbeu.edu.cn (A.M. Zhang).
} 
cavitation near different boundaries as well as its effects on load characteristics and on shock response of structures. The process of cavitation was studied by various researchers by means of theoretical analysis, experiments and numerical modelling. A Taylor flat-plate theory [16] was adopted to analyse the generation of a cavitation near a plate subjected to a plane shock wave. Bleich and Sandler [17] developed a mathematical model of fluids with cavitation and obtained an analytic solution of the velocity of a plate. Li et al. [18] employed a plane shock-wave hypothesis to investigate formation of cavitation caused by an underwater explosion and a shock wave. Clearly, experiment is the most effective method to study cavitation characteristics caused by underwater explosion. Brett et al. [19] conducted a series of experiments to investigate a secondary shock caused by cavitation; the results obtained showed that this loading had a significant effect on deformation of submerged cylinders. Rajendran [20] experimentally studied reloading characteristics and damage of plane plates subjected to a non-contact underwater explosion and found that damage caused by the reloading component was almost equal to that produced by a primary shock pulse. However, the experimental research had various such shortcomings: limited information acquisition, poor operability, etc. With rapid development of computer technology, numerical methods are gradually employed to study cavitation characteristics in the process of underwater explosions. Felippa and DeRuntz [21] used a finite-element analysis for problems of hull cavitation induced by underwater shock. Wardlaw and Luton [22] utilized a coupled GEMINIDYNA hydrocode to investigate formation and collapse of cavitation as well as the target reloading. Sprague and Geers [23] applied a cavitating acoustic finite-element (CAFE) method to simulate the response of a ship to an underwater explosion considering also cavitation. Xie et al. [24-26] used different cavitation models to study the process of large-scale homogeneous unsteady cavitation. Zhang et al. [27] proposed a hybrid method of a fluid spectral element method (SEM) and FEM to analyse secondary loading caused by collapse of cavitation on a spherical shell and stiffened plates. Goncalvès and Charrière [28] implemented a cavitation model in a compressible RANS/Euler solver and studied cavitation dynamics caused by an underwater explosion and bubble collapse. The use of traditional numerical methods is constrained by computational difficulties caused by grid distortion, accurate shock description, moving-interface tracking in highly nonlinear problems of near-field underwater explosions. Besides, due to a high Mach number, compressibility of fluid is significant and should be considered. The coupled method [29-30] of RKDG [31-32], a level-set (LS) method [33-34] and a ghost fluid (GF) method [35-36] was proved to be effective for solution of above problems in compressible fluids. Hence, a hybrid algorithm - RKDG-LS-GF - was employed to analyse a shock load in the fluid. However, this hybrid approach is generally not as good as the finite-element method (FEM) in solving problems of a dynamic response of structures. Therefore, RKDG-LS-GF and FEM are combined here to fully utilize their advantages in simulations of plates subjected to near-field underwater explosions, where the two methods are used respectively for fluid and structural solvers.

In this paper, a coupled algorithm based on RKDG and FEM is used to solve transient fluid-structure interaction (FSI) problems of a shock load for a near-field underwater explosion and a shock response of structures. Besides, formation and collapse of cavitation as well as its reloading effect on structures are studied. First, a cavitation model is introduced into a two-dimensional axisymmetric RKDG-LS-DGF method and a simulation of bubble pulsation in a cylinder is performed. The obtained results are compared with those in the literature to verify the validity of the presented method. Second, a numerical model of a plate subjected to near-field underwater explosion caused by a spherical charge is established by using the developed coupled RKDG-FEM algorithm, and its feasibility is proved through the comparison with experimental data. Third, the pressure and cavitation characteristics for a cylindrical charge are analysed and effects of different parameters thickness of the plate and distance between the charge and the plate - are discussed. Finally, a numerical model of a double plate is developed, and the effect of the distance on load characteristics and structural response is analysed.

\section{Numerical method}

\subsection{Governing equation for Eulerian formulation}

In simulations of an underwater explosion, the explosion gas and the fluid are assumed inviscid, irrotational and compressible. The density, velocity, pressure and total energy per unit volume are denoted as $\rho,(u$, $v, w), P$ and $E$, respectively. The governing equation for a two-dimensional axisymmetric fluid is given by [37]

$U_{t}+\nabla \cdot F(U)=S(U)$,

$$
\text { where } \begin{aligned}
U & =\left[\begin{array}{c}
\rho \\
\rho u \\
\rho v \\
E
\end{array}\right], F=\left[\begin{array}{c}
\rho u \\
\rho u^{2}+p \\
\rho u v \\
u(E+p)
\end{array}\right], S=-\frac{1}{x}\left[\begin{array}{c}
\rho u \\
\rho u^{2} \\
\rho u v \\
u(E+p)
\end{array}\right], E, \\
& =\rho e+\frac{1}{2} \rho\left(u^{2}+v^{2}\right)
\end{aligned}
$$

$e$ is the internal energy per unit mass.

\subsection{Equation of state}

Pressure of detonation products can be presented with the JonesWilins-Lee (JWL) equation of state (EoS) $[29,38]$, expressed as

$P=A\left(1-\frac{\omega \rho}{R_{1} \rho_{0}}\right) e^{-\frac{R_{1} \rho}{\rho_{0}}}+B\left(1-\frac{\omega \rho}{R_{2} \rho_{0}}\right) e^{-\frac{R_{2} \rho}{\rho_{0}}}+\frac{\omega \rho E_{0}}{\rho_{0}}$,

where TNT was chosen as the explosive material; the material constants $A$ and $B$ were set as $3.712 \times 10^{11} \mathrm{~Pa}$ and $3.231 \times 10^{9} \mathrm{~Pa}$, respectively; the constants $R_{1}, R_{2}$ and $\omega$ were taken as $4.15,0.95$ and 0.3 , respectively; the initial density $\rho_{0}$ was $1630 \mathrm{~kg} / \mathrm{m}^{3}$; the specific internal energy $E_{0}$ was $4.29 \times 10^{6} \mathrm{~J} / \mathrm{m}^{3}$.

The Tait EoS $[29,38]$ was applied for water, given by

$P=B\left[\left(\frac{\rho}{\rho_{0}}\right)^{N}-1\right]+A$,

where the constants $A$ and $B$ were taken as $1.0 \times 10^{5} \mathrm{~Pa}$ and $3.31 \times 10^{8}$ Pa, respectively; $N$ was set to 7.15 ; the initial density $\rho_{0}$ was equal to $1000 \mathrm{~kg} / \mathrm{m}^{3}$.

Steel Q235 was used as the material of the plate. The CowperSymonds model [39] was employed for steel, written as

$\sigma_{Y}=\left[1+\left(\frac{\dot{\varepsilon}}{C}\right)^{\frac{1}{P}}\right] \sigma_{0}$,

where $\sigma_{0}$ is the initial yield stress; $\dot{\varepsilon}$ is the strain rate; $C$ and $P$ are parameters for the strain rate, set as $40.4 \mathrm{~s}^{-1}$ and 5 , respectively. The material parameters [40] for Q235 are listed in Table 1.

\subsection{Runge-Kutta discontinuous Galerkin method}

Two-dimensional Euler equations were taken as an example to demonstrate the spatial discretization of the RKDG method which was used to solve the governing equation for the fluid. For a computational

Table 1

Parameters for Q235 [40].

\begin{tabular}{llllll}
\hline $\begin{array}{l}\text { Density } \\
\mathrm{kg} \mathrm{m}^{-3}\end{array}$ & Poisson's ratio & $\begin{array}{l}\text { Young's } \\
\text { modulus } \\
\mathrm{MPa}\end{array}$ & $\begin{array}{l}\text { Yield } \\
\text { stress } \\
\mathrm{MPa}\end{array}$ & $\begin{array}{l}\text { Shear } \\
\text { modulus } \\
\mathrm{MPa}\end{array}$ & $\begin{array}{l}\text { Maximum } \\
\text { plastic strain }\end{array}$ \\
\hline 7850 & 0.3 & $2.1 \times 10^{5}$ & 400 & 250 & 0.23 \\
\hline
\end{tabular}


region $\Omega$ in the fluid, both sides of Eq. (1) were multiplied with a test function $\Phi(x, y)$ and integrated over a cell $K$. Then one has [41-44]

$\int_{K}\left(U_{t}+\nabla \cdot F(U)\right) \Phi(x, y) d \Omega=0$,

$\frac{d}{d t} \int_{K} U \Phi(x, y) d \Omega+\int_{K} \nabla \cdot F(U) \Phi(x, y) d \Omega=0$.

Subsequently, by integrating Eq. (6) by parts, a semi-discrete equation can be obtained, expressed as [41-44]

$\int_{K} \nabla \cdot F(U) \Phi(x, y) d \Omega=\int_{K} \nabla F(U) \Phi(x, y) d \Omega-\int_{K} F(U) \cdot \nabla \Phi(x, y) d \Omega$.

The divergence theorem was used to solve the first term in the righthand side of Eq. (7), given by

$\int_{K} \nabla F(U) \Phi(x, y) d \Omega=\sum_{e \in \partial K} \int_{e} F(U) \cdot \vec{n} \Phi(x, y) d \Gamma$,

where $\vec{n}$ is the unit normal vector of the cell boundary pointing outwards; $\partial K$ is the boundary of element $K$; $e$ is the edge.

By substituting Eqs. (7) and (8) into Eq. (6) one can get [32]

$$
\frac{d}{d t} \int_{K} U \Phi(x, y) d \Omega+\sum_{e \in \partial K} \int_{e} F(U) \cdot \vec{n} \Phi(x, y) d \Gamma-\int_{K} F(U) \cdot \nabla \Phi(x, y) d \Omega
$$$$
=0 \text {. }
$$

The Gaussian quadrature formulas were applied to solve the line and surface integrals in Eq. (9) resulting in [41-44]

$\int_{e} F(U) \cdot \vec{n} \Phi(x, y) d \Gamma \approx \sum_{l=1}^{L} \omega_{l} F\left(U\left(x_{e l}, y_{e l}, t\right)\right) \cdot \vec{n} \Phi\left(x_{e l}, y_{e l}\right)|e|$,
$\int_{K} F(U) \cdot \nabla \Phi(x, y) d \Omega \approx \sum_{j=1}^{M} \omega_{j} F\left(U\left(x_{K j}, y_{K j}, t\right)\right) \cdot \nabla \Phi\left(x_{K j}, y_{K j}\right)|K|$,

where $\omega_{l}$ and $\omega_{\mathrm{j}}$ are weighting coefficients.

The solutions $U$ and $\Phi$ in shock-involved flows must be discontinuous at inter-element boundaries. Hence, the numerical flux $\widehat{F}\left(U\left(x_{e l}, y_{e l}, t\right)\right.$, the exact solutions $U$ and $\Phi$ can respectively replace the flux $F\left(U\left(x_{e b} y_{e v}, t\right)\right.$, the approximate solutions $\widehat{U}$ and $\widehat{\Phi}$. Based on these approximations, Eqs. (10) and (11) are substituted into Eq. (9) [41-44]:

$\frac{d}{d t} \int_{K} \widehat{U} \widehat{\Phi}(x, y) d \Omega+\sum_{e \in \partial K} \sum_{l=1}^{L} \omega_{l} \widehat{F}\left(U\left(x_{e l}, y_{e l}, t\right)\right) \cdot \widehat{\Phi}\left(x_{e l}, y_{e l}\right)|e|$

$-\sum_{j=1}^{M} \omega_{j} \widehat{F}\left(U\left(x_{K j}, y_{K j}, t\right)\right) \cdot \nabla \widehat{\Phi}\left(x_{K j}, y_{K j}\right)|K|=0$

where the numerical flux $\widehat{F}\left(U\left(x_{e l}, y_{e l}, t\right)\right.$ is substituted with the LaxFriedrichs flux, given by

$\widehat{F}\left(U\left(x_{e l}, y_{e l}, t\right)\right)=\frac{1}{2}\left(\widehat{F}\left(U^{-}\left(x_{e l}, y_{e l}, t\right)\right)+\widehat{F}\left(U^{+}\left(x_{e l}, y_{e l}, t\right)\right)\right)$,

$-\frac{\alpha}{2}\left(U^{+}\left(x_{e l}, y_{e l}, t\right)-U^{-}\left(x_{e l}, y_{e l}, t\right)\right)$

where $\alpha$ is the maximum eigenvalue of the Jacobian matrix $\partial F(U) / \partial U$. The approximate solution $\widehat{U}(x, y, t)$ is obtained inside the quadrilateral element $\left[x_{i-1 / 2}, x_{i+1 / 2}\right] \times\left[y_{j-1 / 2}, y_{j+1 / 2}\right]$, and is written as

$\widehat{U}(x, y, t)=\bar{U}(t)+U_{x}(t) \xi_{i}(x)+U_{y}(t) \eta_{j}(y)+U_{x y}(t) \xi_{i}(x) \eta_{j}(y)$

$+U_{x x}(t)\left(\xi_{i}(x)^{2}-\frac{1}{3}\right)+U_{y y}(t)\left(\eta_{j}(y)^{2}-\frac{1}{3}\right)$

where $\quad \xi_{i}(x)=\frac{x-x_{i}}{\Delta x_{i} / 2}, \quad \eta_{j}(y)=\frac{y-y_{j}}{\Delta y_{j} / 2}, \quad \Delta x_{i}=x_{i+1 / 2}-x_{i-1 / 2} \quad$ and $\Delta y_{j}=y_{j+1 / 2}-y_{j-1 / 2}$.

Substituting Eqs. (13) and (14) into Eq. (12), and the semidiscrete formulation can be given as

$U_{t}=R(U)$,

where $R(U)$ is the discrete operator of spatial derivatives.

Finally, the discrete difference scheme was obtained by using the third-order total variation diminishing (TVD) of the Runge-Kutta time discretization method for Eq. (15), expressed as [32]

$U^{(1)}=U^{(n)}+\Delta t R\left(U^{(n)}\right)$,

$U^{(2)}=\frac{3}{4} U^{(n)}+\frac{1}{4}\left(U^{(1)}+\Delta t R\left(U^{(1)}\right)\right)$,

$U^{n+1}=\frac{1}{3} U^{(n)}+\frac{2}{3}\left(U^{(2)}+\Delta t R\left(U^{(2)}\right)\right)$.

In addition, the above RKDG method was coupled with LS and GF methods to capture, and deal with, the moving interfaces of the multiphase flow. The detailed coupling procedure of the above methods can be found in [29] and [41].

\subsection{Cut-off cavitation model}

A cavitation model should be employed to update a relationship between pressure and density of fluid when the former is lower than the saturated-vapor pressure $P_{\text {sat }}$. A high-applicability cut-off cavitation model has little effect on computational accuracy of a pressure peak in the high-pressure fluid. Hence, this model was utilized in this paper, given by [29]

$P= \begin{cases}B\left[\left(\frac{\rho}{\rho_{0}}\right)^{N}-1\right]+A, & \rho>\rho_{\text {sat }}, \\ P_{\text {sat }}, & \rho \leq \rho_{\text {sat }}\end{cases}$

where $\rho_{\text {sat }}$ is the density when the pressure is equal to the saturated vapor pressure $P_{\text {sat }}$.

This cavitation model was introduced into the two-dimensional axisymmetric RKDG-LS-GF method. In order to verify the effectiveness of the presented method, a numerical model of an ideal-gas bubble pulsation in a water-filled rigid cylinder was developed and its results were compared with those in [24]. The magnitudes of diameter and length of the cylinder were $0.0889 \mathrm{~m}$ and $0.2286 \mathrm{~m}$, respectively. The bubble with a diameter of $0.03 \mathrm{~m}$ was placed at the centre of the cylinder. The bubble had an initial density of $1770 \mathrm{~kg} / \mathrm{m}^{3}$ and pressure $2 \times 10^{9} \mathrm{~Pa}$. As for the surrounding water, its density and pressure were equal to $1000 \mathrm{~kg} / \mathrm{m}^{3}$ and $1 \times 10^{5} \mathrm{~Pa}$, respectively. The levels of pressure of the gas and the water were obtained from the state equations of ideal gas and Tait. The saturated vapor pressure $P_{\text {sat }}$ in the cut-off cavitation model was set to $5000 \mathrm{~Pa}$. The comparison of results for pressure distributions in the fluid are shown in Fig. 1, where the colored (right) and the black-and-white (left) images correspond to results obtained by our method and in [24], respectively. A spherical shock wave (SW1) was generated during an expansion stage of the bubble. After this shock wave arrived at the rigid wall, a reflected shock wave (RSW1) was produced and propagated in the fluid. When RSW1 reached the bubble, a rarefaction wave (RRW1) was generated (Fig. 1(a)) because impedance of the water was higher than that of the bubble. Subsequently, a rarefaction wave (RRW2) was produced after RRW1 arrived at the rigid wall. Due to the superimposition of these two rarefaction waves, a cavitation with low pressure was formed (Fig. 1(b)). With the propagation of complex waves in the fluid, the cavitation gradually collapsed (Figs. 1(c) and (d)). The numerical results obtained for the pressure distribution show a good agreement with those in [24], proving the validity of our numerical method.

A further comparison of pressure evolution at the centre of the rigid cylinder is presented in Fig. 2. Two pressure peaks were caused by the direct shock wave and the collapse of the cavitation, respectively. Obviously, our results agreed well with those in [24]. This indicates that the developed RKDG-LS-GF method for the cavitation model can effectively simulate the processes of formation and collapse of cavitation.

\subsection{Coupled RKDG-FEM}

In FSI problems, a hybrid mesh is generally used when the fluid and the structure domains are, respectively, analysed employing the Euler 

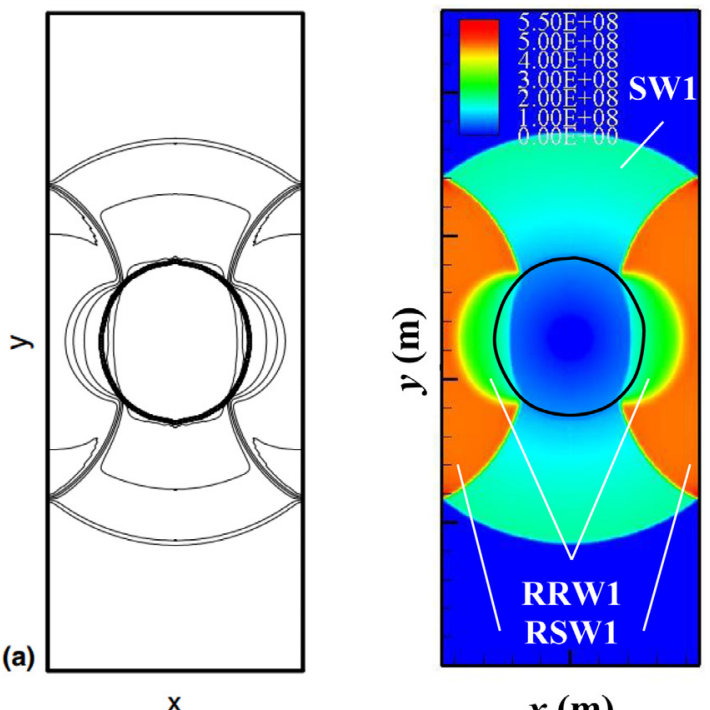

$x(\mathbf{m})$

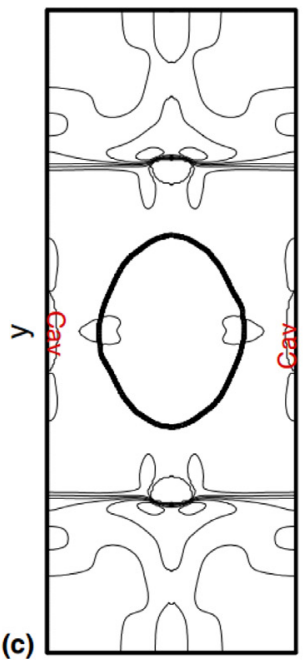

$\mathbf{X}$

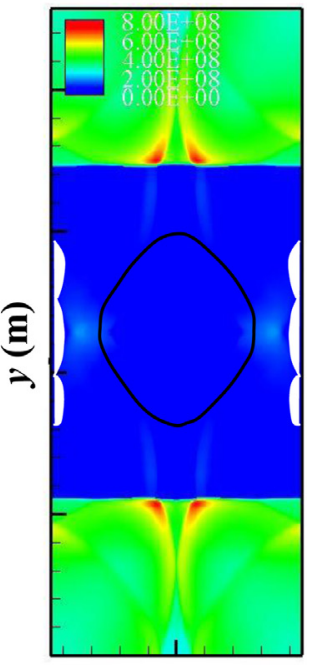

$x(\mathbf{m})$
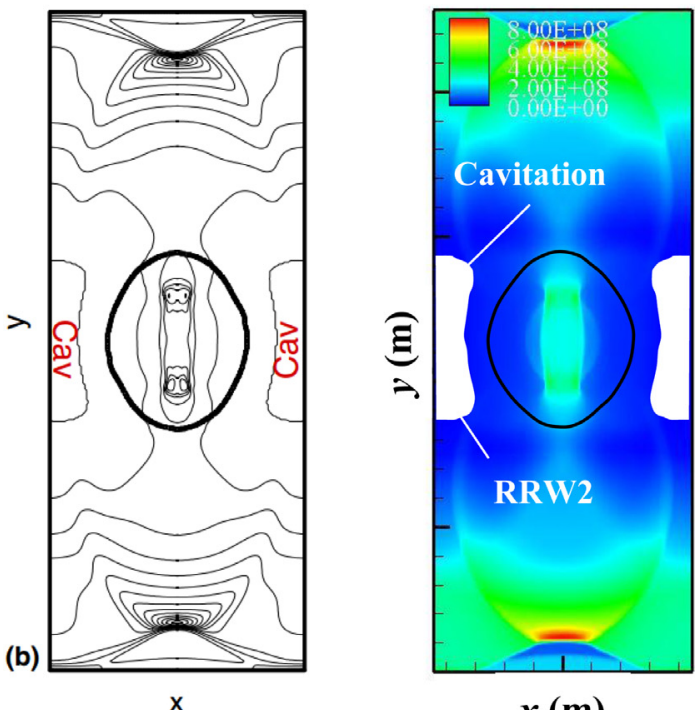

$\boldsymbol{x}(\mathbf{m})$

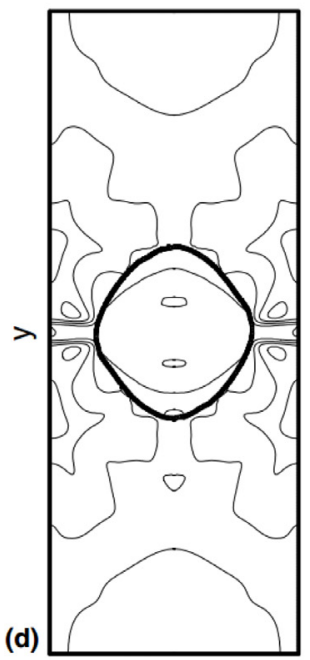

$\mathbf{x}$

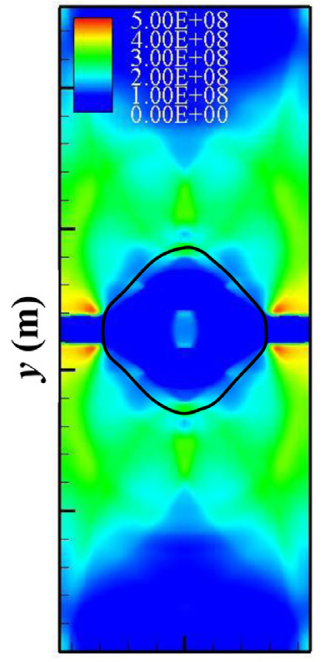

$x(\mathbf{m})$

Fig. 1. Comparison of results for pressure distribution obtained with our method (right) and Ref. [24] (left): (a) $30 \mu s$, (b) $60 \mu s$, (c) $90 \mu s$, (d) $120 \mu s$.

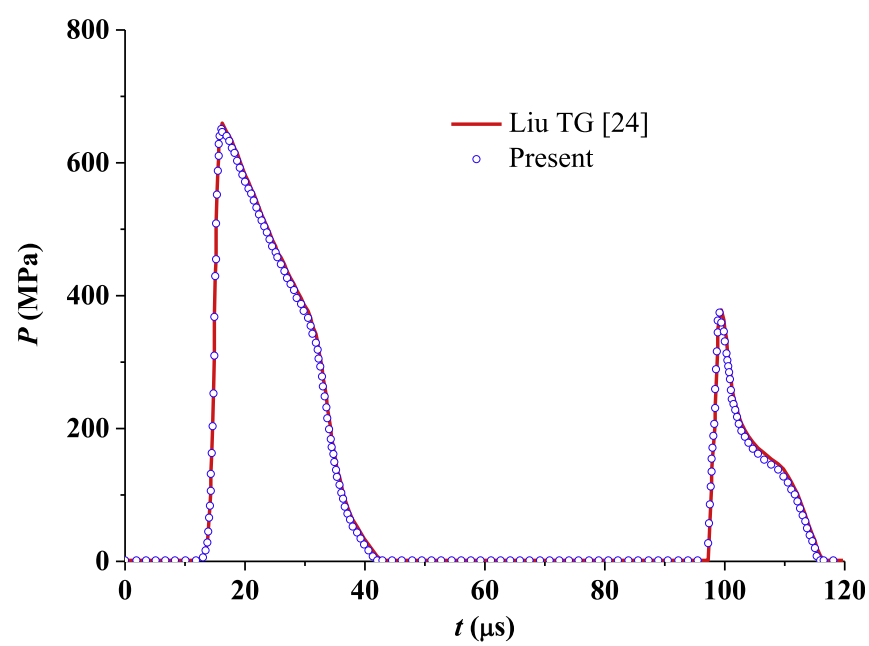

Fig. 2. Evolultion of pressure at centre of rigid wall. and Lagrange formulations, resulting in a distorted mesh for an elastoplastic structure under large deformations. In this paper, a coupled RKDG-FEM method $[29,31]$ is proposed to overcome this limitation and study pressure and cavitation characteristics of a near-field underwater explosion of cylindrical charges near single/double plates. The detailed coupled procedure was implemented with the following steps:

(1) The pressure at the fluid-structure interface was obtained with the fluid solver - the RKDG method.

(2) FE software ABAQUS was used to assess loads employing a VDLOAD FORTRAN subroutine. The level of pressure obtained with the fluid solver was regarded as the initial load for the structural solver in ABAQUS. Subsequently, material deformation and velocities at the interface were updated for the structural solver.

(3) The updated coordinates and velocities were used as the new boundary conditions in the fluid solver at the next step.

The RKDG and FEM methods were respectively utilized to solve the governing equation for the fluid and to analyse a structural response. As for the fluid-structure interaction, the motion equations and dynamic continuity conditions are given by 


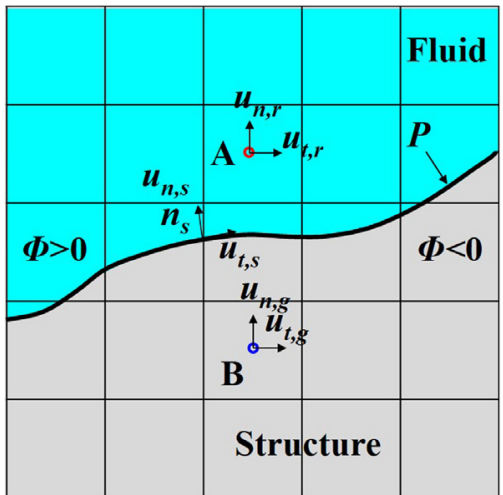

Fig. 3. Fluid-structure interaction treatment.

$u_{n, f}=u_{n, s}$,

$P=F$,

where $u_{n, f}$ and $u_{n, s}$ are the normal velocities at the surface of the structure in the fulid and structure domains, respectively; $P$ and $F$ are pressures at the surface of these two domains, respectively.

The level set method (LSM) was applied to capture the interaction, with function values $\Phi$ setting to values lower and higher than 0 in the structure and the fluid, respectively. The extrapolation for the ghost fluid method (GFM) was used for boundary conditions at the interaction. The real and ghost cells were denoted as A and B (Fig. 3), respectively. The boundary conditions for cell B can be obtained in terms of physical quantities $\left(\rho_{g}, u_{n, g}, u_{t, g}, P_{g}\right)$ for the GFM, where $u_{n, g}$ and $u_{t, g}$ were the normal and tangential velocities. According to the kinematical boundary condition, the normal velocity $u_{n, g}$ was obtained by the linear extrapolation. This approach has an advantage in solving problems with fluid-structure interaction because a mesh in the fluid does not need to match completely with that in the structure, allowing for full de-coupling between nodes in two domains. For the fluid domain, the profile and the velocity in the structure were used; as for the structure one, the shock load in the fluid was needed. Pressure $P$ got with the fluid solver was regarded as the initial load for the structure solver in ABAQUS software [45]. The equation for the structural response was given by [45]

$$
\begin{aligned}
\int_{V} \kappa \varepsilon: \sigma d V+ & \int_{V} \alpha_{c} \rho \kappa \mathbf{u} \cdot \dot{\mathbf{u}} d V+\int_{V} \rho \kappa \mathbf{u} \cdot \ddot{\mathbf{u}} d V \\
& +\int_{S_{f s}} P \kappa \mathbf{u} \cdot \mathbf{n} d S-\int_{S_{t}} \kappa \mathbf{u} \cdot \mathbf{t} d S=0,
\end{aligned}
$$

where $\kappa$ is the variation; $\varepsilon$ and $\boldsymbol{\sigma}$ are the strain and stress tensors; $\alpha_{c}$ is the damping factor; $\mathbf{u}, \dot{\mathbf{u}}$ and $\ddot{\mathbf{u}}$ are the displacement, velocity and acceleration, respectively; $\mathbf{n}$ is the normal vector.

The coupling scheme for the used methods is presented in Fig. 4. First, the pressure at fluid and the structure interface was obtained with the RKDG method for the fluid domain. Second, this pressure was used as the load for the structure domain and, subsequently, the velocity at the interaction was updated. Third, this updated velocity was used in the fluid solver. The coordinates of the structure were updated by using the LSM. Finally, the simulations returned to the first step, and the physical quantities in the fluid were updated with the GFM.
3. Pressure characteristics and cavitation effects of underwater explosion near plates

A strongly discontinuous shock wave can be generated in fluid after an explosive is detonated. Rarefaction waves can be produced as a result of repeated reflection of this shock wave between the bubble and the structure, resulting in generation of a cavitated region with low pressure. This cavitation has a significant effect on load characteristics and on structural response. Hence, after the presented approach - the RKDG method with the cut-off cavitation model - was successfully verified, it was used for the fluid domain and the analysis of pressure and cavitation characteristics. As for the structure domain, the FEM was employed to analyse dynamic response of structures. A two-dimensional axisymmetric RKDG-FEM model of a plate subjected to a nearfield underwater explosion of a spherical charge was first developed; the obtained results for deflection distribution were compared with those of experimental data in order to verify the validity of the coupled RKDG-FEM method in the next section.

\subsection{Numerical verification for coupled RKDG and FEM}

To verify the developed approach, an initial study was implemented focused on the available experimental evidence. A numerical model of a circular plate subjected to a near-field underwater explosion was developed following the experiment in [46], and its schematic is shown in Fig. 5 (the symmetry axis (z) was vertical). The modelled steel plate had a radius $R=53 \mathrm{~mm}$ and thickness $t=1.9 \mathrm{~mm}$. The mass $m$ of the spherical charge was $5 \mathrm{~g}$. The distance between the centre of the charge and the plate was about $25 \mathrm{~mm}$. Numerical results obtained with the developed scheme were compared with the experimental data.

A comparison of the results for the deformation of the plate is presented in Fig. 6. Apparently, the near-field underwater explosion resulted in large plastic deformation of the plate. It was found that the shapes of the deformed plate obtained with the coupled RKDG-FEM method and the experiment were close, with the error for the maximum deflection lower than $2 \%$, proving the effectiveness of the presented method. A further comparison of deflection distribution of the plate is presented in Fig. 7, demonstrating a decline of the deflection magnitude with the distance form its centre. The good agreement of our numerical results with the experimental data verified the validity of the developed RKDG-FEM method, indicating that this numerical method can solve problems of a shock response of structures to near-field underwater explosions effectively.

\subsection{Analysis of single plate}

After this validation of the presented algorithm, a two-dimensional axisymmetric RKDG-FEM model of a plate subjected to a near-field underwater explosion of a cylindrical charge was developed; pressure and cavitation characteristics of the process were analysed.

\subsubsection{Numerical model}

A schematic of the problem for the plate subjected to the near-field underwater explosion, with a coordinate system is shown in Fig. 8, with the centre of the plate coinciding with its origin point (the symmetry axis (z) was vertical). The steel plate had a radius $R=1 \mathrm{~m}$ and

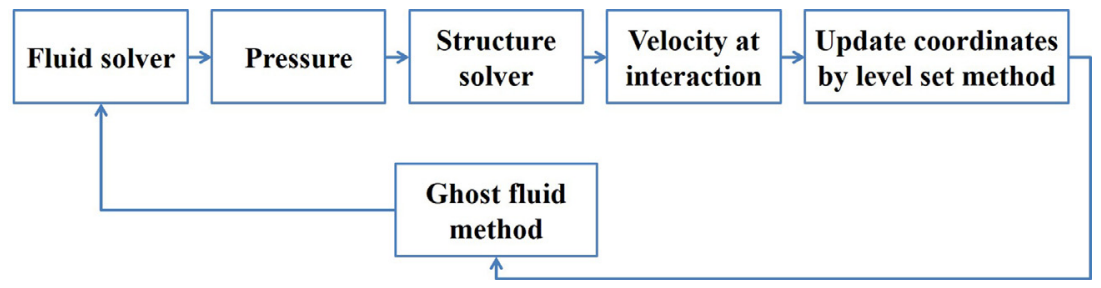

Fig. 4. Procedure for fluid-structure interaction treatment. 


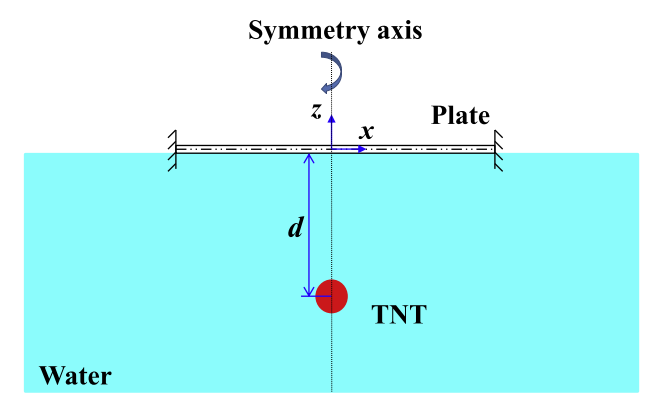

Fig. 5. Model of plate subjected to near-field underwater explosion of spherical charge.

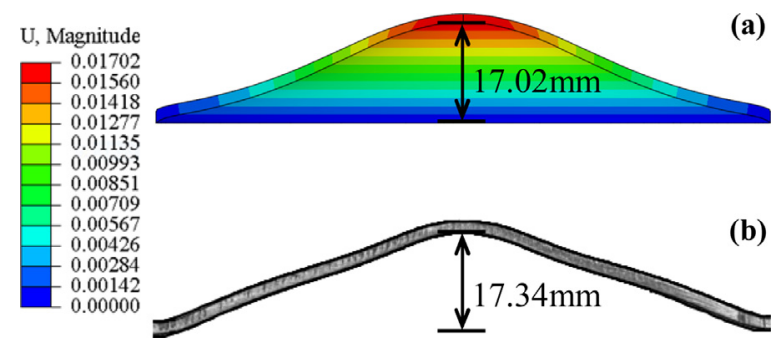

Fig. 6. Numerical (a) and experimental [46] (b) results for shape of deformed plate after near-field underwater explosion of spherical charge.

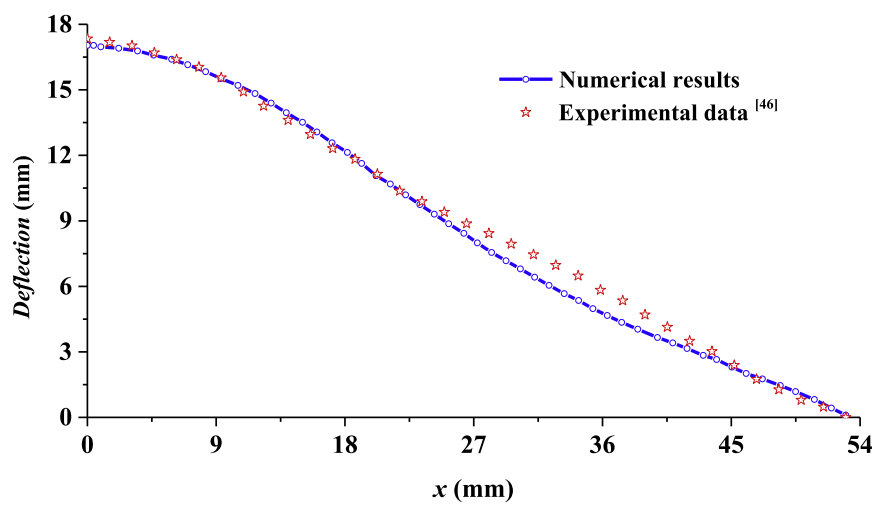

Fig. 7. Deflection distribution for plate after near-field underwater explosion of spherical charge.

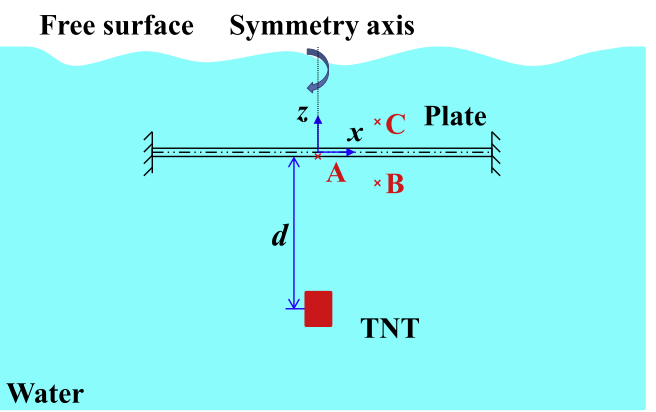

Fig. 8. Model of plate subjected to near-field underwater explosion of cylindrical charge.

thickness $t=0.016 \mathrm{~m}$. A cylindrical charge was used in this model, with a radius of $0.1 \mathrm{~m}$ and height of $0.2 \mathrm{~m}$. The distance between the centre of the charge and the plate was about $0.5 \mathrm{~m}$. Coordinates for test points A, B and C were $(0,-0.008),(0.3,-0.2)$ and $(0.3,0.2)$, respectively. The mesh sizes of the plate along its length and the fluid were $0.01 \mathrm{~m}$ and $0.005 \mathrm{~m}$, respectively. Continuum elements were used to model the FE plate, with 4 and 100 elements through its thickness and
Table 2

Cases for single-plate simulation.

\begin{tabular}{llll}
\hline Case & Rigid wall/ Elastic-plastic plate & Distance $d$ & Thickness of plate $h$ \\
\hline Case 1 & Rigid wall & $0.4 \mathrm{~m}$ & - \\
Case 2 & Elastic-plastic plate & $0.4 \mathrm{~m}$ & $0.016 \mathrm{~m}$ \\
Case 3 & Elastic-plastic plate & $0.3 \mathrm{~m}$ & $0.016 \mathrm{~m}$ \\
Case 4 & Elastic-plastic plate & $0.5 \mathrm{~m}$ & $0.016 \mathrm{~m}$ \\
Case 5 & Elasktic-plastic plate & $0.4 \mathrm{~m}$ & $0.020 \mathrm{~m}$ \\
Case 6 & Elastic-plastic plate & $0.4 \mathrm{~m}$ & $0.024 \mathrm{~m}$ \\
\hline
\end{tabular}

along its length, respectively. Cases for the single-plate simulations discussed in this section are listed in Table 2.

\subsubsection{Shock wave propagation and cavitation characteristics}

The character of pressure distribution, deflections and schlieren images are shown in Fig. 9 for Case 2. The explosion products expanded rapidly after the cylindrical charge was detonated. A high-pressure shock wave (SW1) and a rarefaction wave (RW1) were generated in the water and the explosion products (Fig. 9(a)), respectively. This figure also shows that the explosion products retained the initial cylindrical shape right after the detonation, evolving subsequently into a spherical shape (Fig. 9(b)). After SW1 arrived at the plate, a reflected shock wave (RSW1) was generated towards the explosion products since impedance of steel was higher than that of water (Fig. 9(b)). These two shock waves caused deformation of the plate. At the same time, a transmitted shock wave (TSW1) was produced and propagated behind the plate. When RSW1 reached the explosion products, a reflected rarefaction wave (RRW1) was generated (Fig. 9(c)) as a result of higher impedance of water than that of the explosion products. Subsequently, RRW1 arrived at the plate (Fig. 9(d)), with another rarefaction wave (RRW2) reflected in the water between the plate and the bubble. The superposition of these two rarefaction waves resulted in a cavitation with low pressure. Additionally, it can be found that the propagation velocity of the transmitted shock wave in the bubble (TSW2) was lower than that of RSW1 in the water. With propagation of rarefaction waves in the fluid, the cavitation grew and collapsed, disappeared along the bottom surface of the plate (Figs. 9(e) and 9(f)), with a large deformation of the plate. Additionally, a triple point and a Mach stem can be observed in Fig. 9(f).

\subsubsection{Pressure characteristics}

After the analysis of shock-wave propagation, pressure evolution with time at test points $\mathrm{B}$ and $\mathrm{C}$ in the fluid was studied first and is shown in Fig. 10. The incident shock wave (SW1) first reached test point B, resulting in the first peak. Subsequently, RSW1 was produced and propagated in the water below the plate, causing the second peak. Besides, TSW1 was also generated above the plate, leading to the first peak at point C. Additionally, it can be seen that the peak caused by TSW1 was lower than that of SW1 and RSW1.

Subsequently, a comparison of pressure evolution at the centre of either a rigid wall or the elastic-plastic plate was performed; the history curves of pressure at point A for respectively Cases 1 and 2 are shown in Fig. 11. After the incident shock wave arrived at the plate/rigid wall, the pressure level instantaneously peaked at about $2.3 \mathrm{GPa}$ and $2.1 \mathrm{GPa}$ in Cases 1 and 2, respectively. The level of pressure in Case 2 was lower because the elastic-plastic plate moved under the effect of SW1, and both RSW1 and TSW1 were generated while only RSW1 was produced in Case 1. After that, the pressure diminished drastically. The decrease rate in Case 2 was higher due to the generation of TSW1. Subsequently, the low-pressure cavitation was first formed at the bottom surface of the plate as a result of the superposition of RRW1 and RRW2 and, then, gradually collapsed, causing the secondary loading. Obviously, the cavitation in Case 2 disappeared faster than that in Case 1 while the secondary peak was slightly lower.

After that, the structural response of the plate was studied; 

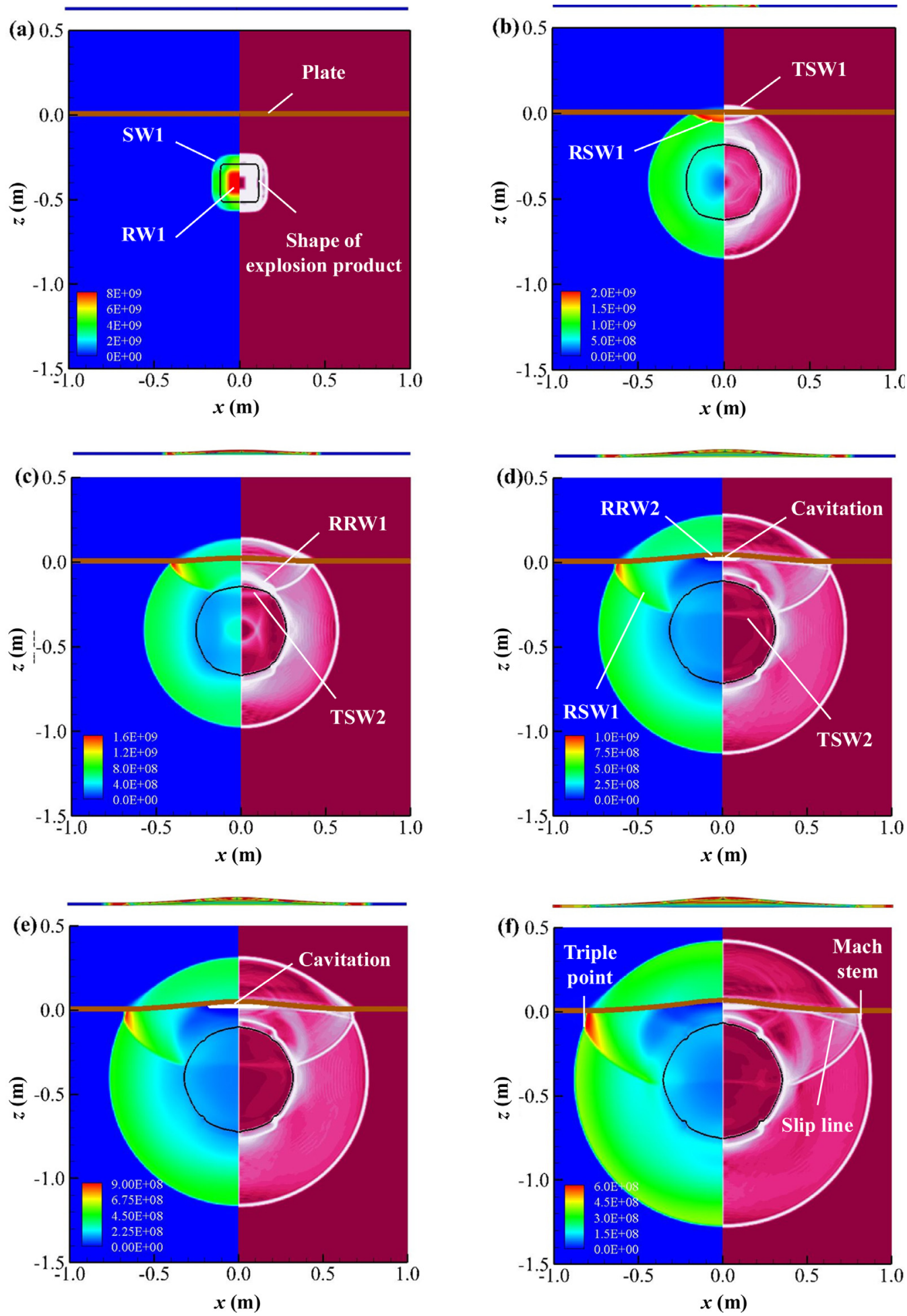

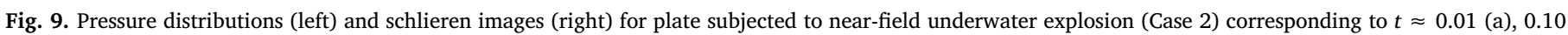
(b), 0.16 (c), 0.23 (d), 0.24 (e) and 0.30 (f) $\mathrm{ms}$.

evolution of velocity and displacement at point A are illustrated in Fig. 12. Under the effect of SW1, the plate moved upwards. Its velocity increased rapidly to about $350 \mathrm{~m} / \mathrm{s}$ and gradually decreased as a result of the pressure decay of and formation of cavitation. The displacement at point A tended to increase steadily with time.

The comparison results for distributions of von Mises stress and equivalent plastic strain of the plate at its top and bottom surfaces are presented in Fig. 13. At about $0.086 \mathrm{~ms}$, the plate was impacted by
SW1. The level of von Mises stress at the bottom surface was higher (Figs. 13(a-1)) and plastic deformation occurred, compared to the top surface (Figs. 13(a-2)). With propagation of the shock wave, the plate continued to deform. The magnitude of von Mises stress at the bottom surface was lower than that at the top one, while its equivalent plastic strain was higher at about $0.15 \mathrm{~ms}$ (Figs. 13(b-1) and (b-2)). The whole plate was shocked at about $0.3 \mathrm{~ms}$; the levels of von Mises stress and equivalent plastic strain at the bottom surface were both lower 


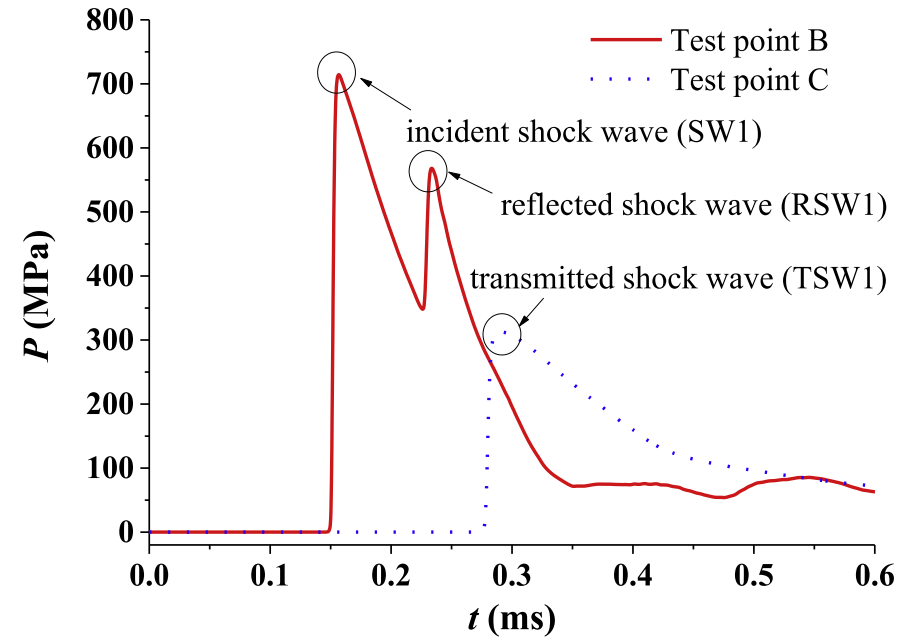

Fig. 10. Evolution of pressure at points B and C (see Fig. 8).

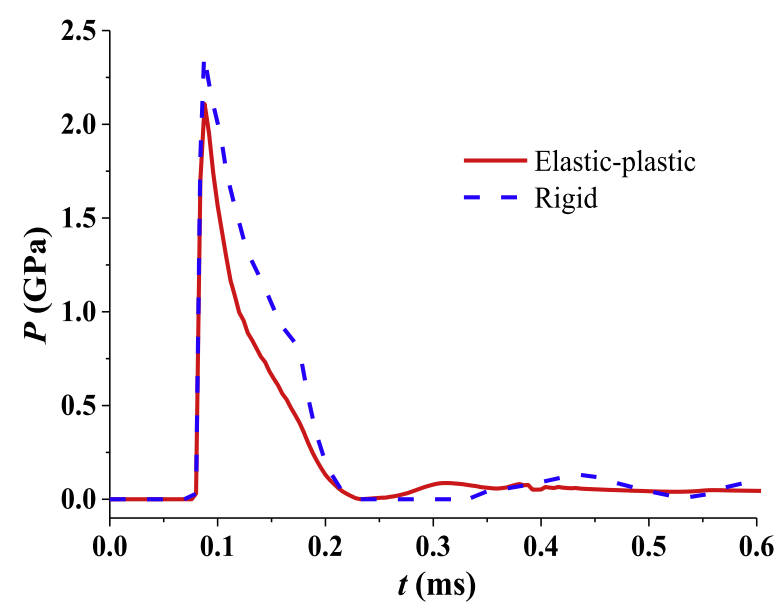

Fig. 11. Evolution of pressure at centre of elastic-plastic plate and rigid wall.

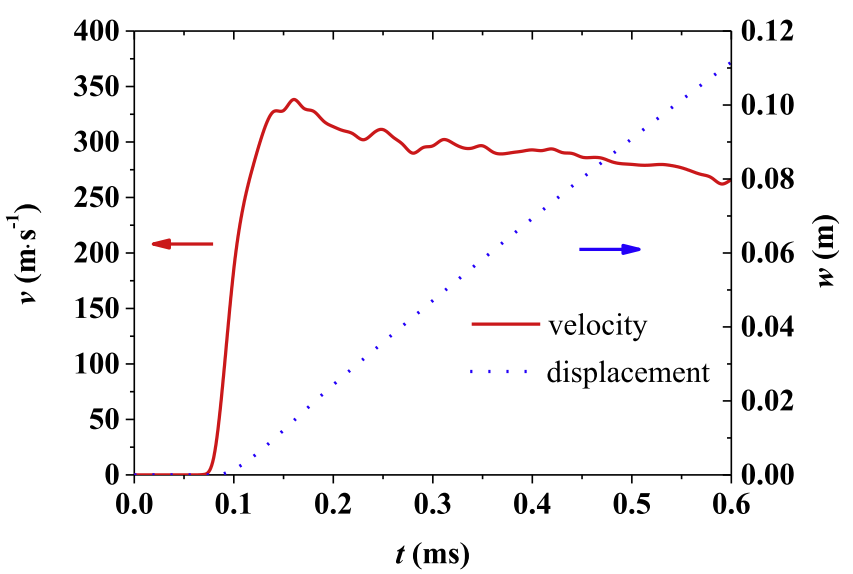

Fig. 12. Evolutions of velocity and displacement at centre of single plate.

(Figs. 13(c-1) and (c-2)). The equivalent plastic strains at the bottom and top surfaces were about 0.029 and 0.008 , indicating that large deformations of the plate were caused.

\subsubsection{Effects of different parameters on load characteristics}

(1) Effect of distance

Next, the effect of distance on load characteristics and a structural response was analysed. The distance $d$ between the charge and the plate ranged from $0.3 \mathrm{~m}$ (Case 3), $0.4 \mathrm{~m}$ (Case 2) to $0.5 \mathrm{~m}$ (Case 4). Other parameters of the model were the same as in Case 2 .

Evolution of pressure at the bottom surface of the plate is shown in Fig. 14. The comparison of the three cases demonstrates that the pressure peak and the decrease rate in Case $3(d=0.3 \mathrm{~m})$ was higher than that in Cases $2(d=0.4 \mathrm{~m})$ and $4(d=0.5 \mathrm{~m})$. Besides, other than Case $3(d=0.3 \mathrm{~m})$, due to the superposition of rarefaction waves, the pressure values in Cases $2(d=0.4 \mathrm{~m})$ and $4(d=0.5 \mathrm{~m})$ were lower than the saturated-vapor pressure and a cavitation was generated as a result. The reason for this phenomenon is the fact that wave strength diminished with the increase in the distance. It indicates that the cavitation would not be formed at a closer range.

Evolutions of velocity and displacement at the centre of plate are illustrated in Fig. 15. The three cases shared a trend for velocity - increasing first and then decreasing; while the level of displacement increased continuously. The maximum deflection in the three cases reached about $0.15 \mathrm{~m}, 0.11 \mathrm{~m}$ and $0.08 \mathrm{~m}$, respectively, while the velocity peaked at about $450 \mathrm{~m} / \mathrm{s}, 350 \mathrm{~m} / \mathrm{s}$ and $250 \mathrm{~m} / \mathrm{s}$. It can be found from the comparison of the obtained results that a decrease in the distance resulted in the increased deformation and velocity; additionally, the levels of velocity and displacement began to increase earlier.

\section{(2) Effect of plate thickness}

In this section, the effect of plate thickness on load characteristics is discussed, with thicknesses values $h$ of $0.016 \mathrm{~m}$ (Case 2), $0.020 \mathrm{~m}$ (Case 5) and $0.024 \mathrm{~m}$ (Case 6) studied; other parameters were the same as in Case 2.

A comparison of pressure evolution with time at the centre of the plate is presented in Fig. 16. The initial tendencies for the three cases were similar - increasing to the peak at first and then diminishing. The pressure peak value was higher for the increase in thickness because of the lower intensity of the transmitted shock wave. Besides, the larger the thickness, the faster the cavitation process was formed. Additionally, the duration of the cavitation collapse was longer and the secondary-peak value was higher. The reason for this phenomenon is the fact that the intensities of the two rarefaction waves reflected, respectively, by the bubble and the plate were higher with the thickness increase.

Evolutions of velocity and displacement at the centre of the plate are shown in Fig. 17. These parameters had a similar tendency - the level of velocity rapidly increased and stayed nearly steady eventually, while the displacement increased continuously with time. It can be found from the comparison of the obtained results that the levels of velocity and displacement diminished with the thickness increase.

\subsection{Analysis of double plates}

\subsubsection{Numerical model}

After the discussion of cases of the single plate, a two-dimensional axisymmetric RKDG-FEM model with a vertical symmetry axis ( $z$ ) of double plates subjected to a near-field underwater explosion was developed (Fig. 18). Parameters of the charge and the plates were the same as in Section 3.2.1. The distance between the two plates was $0.1 \mathrm{~m}$. The centre of the lower plate coincided with the origin point. Test points A, B, C and D were placed at $(0,-0.008),(0.52,-0.05)$, $(0.52,0.05)$ and $(0.52,0.15)$, respectively.

\subsubsection{Shock wave propagation and cavitation characteristics}

The character of pressure distribution and schlieren images are presented in Fig. 19 (the upper and lower plates are denoted Plates II and I, respectively). The water domain is divided into three sub-domains - K, L and M - see Fig. 19(a). A high-pressure shock wave (SW1) was generated in sub-domain $\mathrm{M}$ after the cylindrical charge was 
Bottom surface
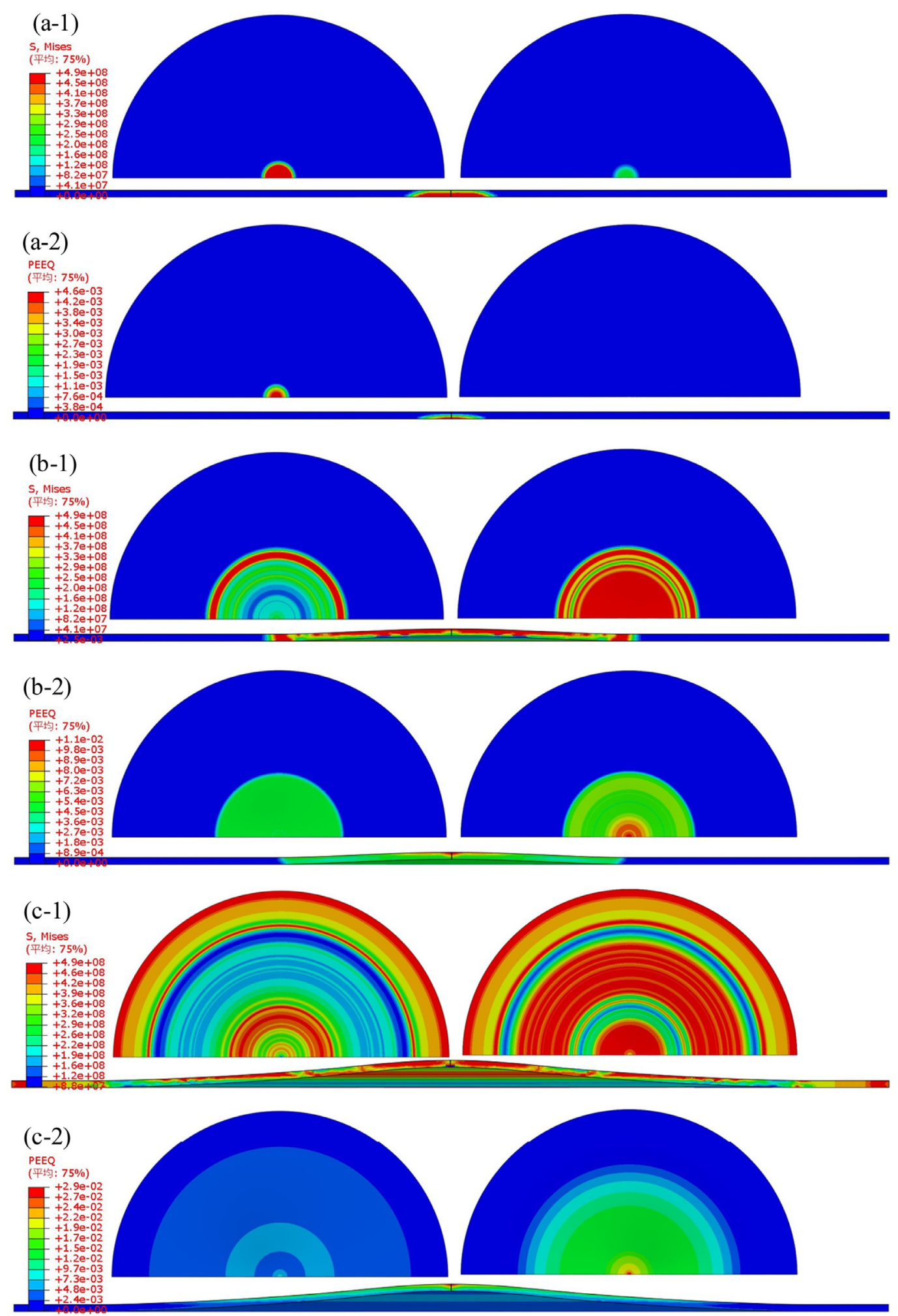

Fig. 13. Evolutions of von Mises stress (1) and equivalent plastic strain (2) at top (right) and bottom (left) surfaces corresponding to $t \approx 0.086$ (a), 0.150 (b) and 0.300 (c) $\mathrm{ms}$.

detonated. When SW1 reached Plate I, not only a reflected shock wave (RSW1) was generated in sub-domain M but also a transmitted shock wave (TSW1) was produced in sub-domain L. Subsequently, TSW1 arrived at Plate II, and as a result, a reflected shock wave (RSW2) and a transmitted shock wave (TSW2) were generated in sub-domains L and $\mathrm{K}$, respectively. As RSW2 reached Plate I, a reflected shock wave (RSW3) and a transmitted shock wave (TSW3) were produced in subdomains L and M (Fig. 19(b)), respectively. Obviously, a complex wave 


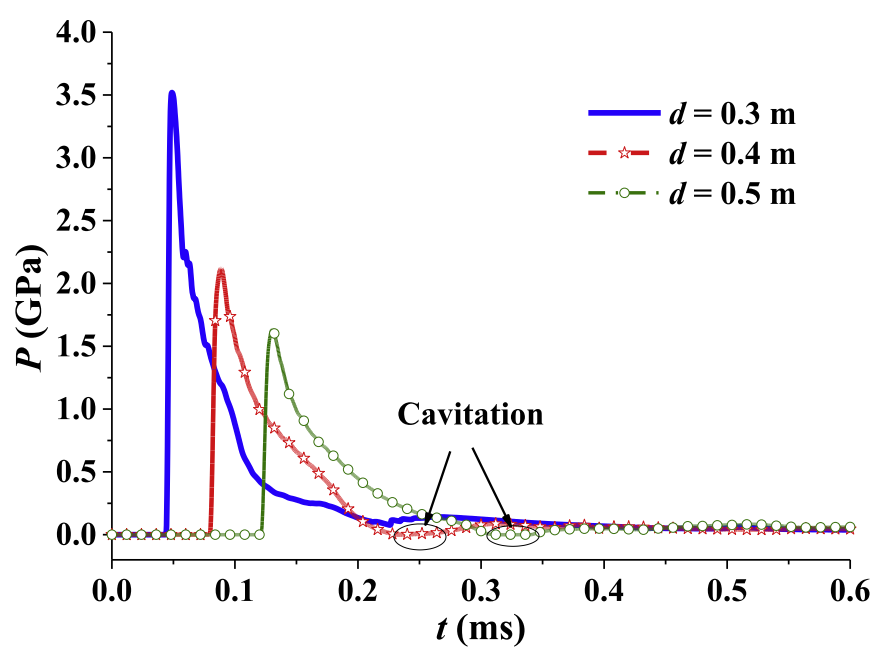

Fig. 14. Evolution of pressure at centre of plate's bottom face for different distances.

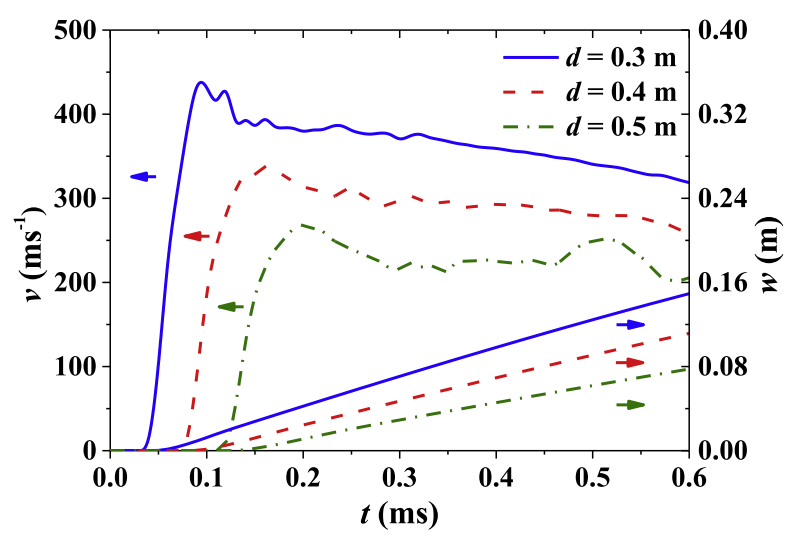

Fig. 15. Evolutions of velocity and displacement at centre of plate for different distances.

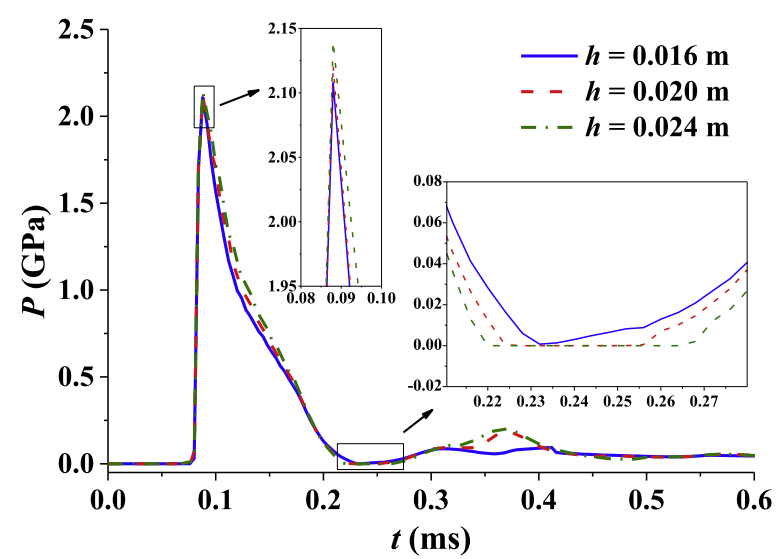

Fig. 16. Evolution of pressure at centre of plate for different thicknesses.

structure was formed by propagation of these waves. After RSW1 arrived at the bubble, a rarefaction wave (RRW1) was reflected in subdomain M. When RRW1 reached Plate I, both reflected (RRW2) and transmitted (TRW1) rarefaction waves were produced in sub-domains $\mathrm{M}$ and $\mathrm{L}$. The level of pressure of the reflected waves decayed quickly due to the repeated reflections and transmissions due to the two plates. A cavitation zone was formed (Fig. 19(c)) due to the superposition of the rarefaction and shock waves. As TRW1 impacted Plate II, a reflected rarefaction wave (RRW3) and a transmitted rarefaction wave (TRW2)

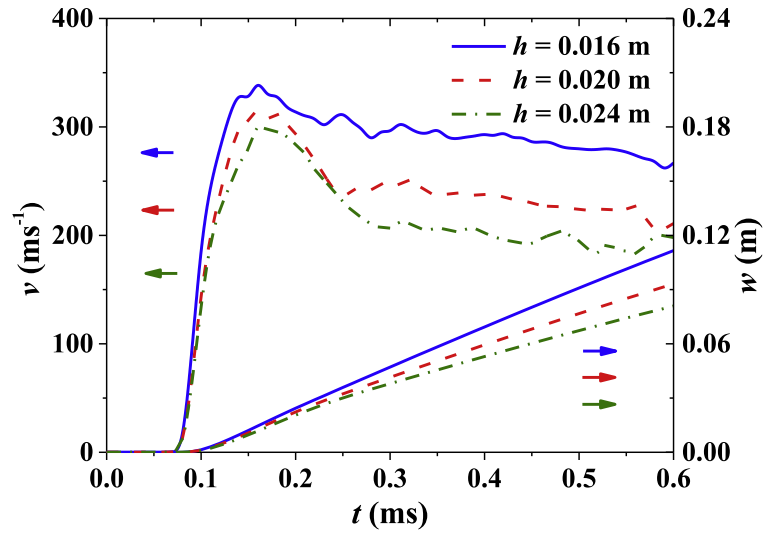

Fig. 17. Evolutions of velocity and displacement at centre of plate for different thicknesses.

Free surface Symmetry axis

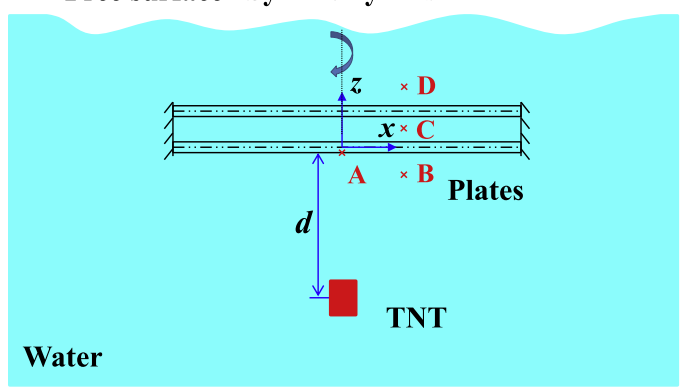

Fig. 18. Model of double plate subjected to near-field underwater explosion of cylindrical charge.

were generated (Fig. 19(d)). The superposition of RRW2 and RRW3 enlarged the cavitation region. Apparently, the wave structure for the case of double plates was more complex than that for the single plate.

\subsubsection{Pressure characteristics}

After the analysis of shock-wave propagation, pressure evolution with time at test points B, C and D in the fluid was studied and is presented in Fig. 20. As SW1 arrived at point B, the pressure peaked at about $1.1 \mathrm{GPa}$ and then decreased exponentially. After RSW1 reached this point, a second peak, lower than the first one, was formed. Subsequently, TSW1 and RSW2 were generated and propagated successively in sub-domain L, causing the first and second increases of the levels of pressure at point $\mathrm{C}$. The pressure peaked at about $750 \mathrm{MPa}$ and then declined. The cavitation zone was formed when the rarefaction waves propagated in sub-domain $\mathrm{L}$ and subsequently collapsed, resulting in the secondary loading. As for point D, the pressure peak caused by the arrival of TSW1 was much lower than that at points B and C.

Evolution of pressure at the centre of Plates I and II is illustrated in Fig. 21. The pressure increased rapidly when SW1 reached Plate I. Multi-peaks can be found in Fig. 21 due to repeated reflections and transmissions of shock and rarefaction waves. As for Plate II, the first and second peaks were caused by TSW1 and RSW3, respectively.

A further comparison of pressure distributions at Plates I and II was studied and is presented in Fig. 22. For Plate I, the pressure distribution at the early stage was similar to that of the single plate while it was more complex due to the superpositions of repeatedly reflected waves at later stages (Fig. 22(a)). As for Plate II, the pressure rapidly increased under the effect of TRW1 (Fig. 22(b)). The pressure-peak value moved gradually sideways along this plate. After RSW3 reached it, the second peak was formed and moved sideways as well. The peak values decreased due to repeated reflections. 

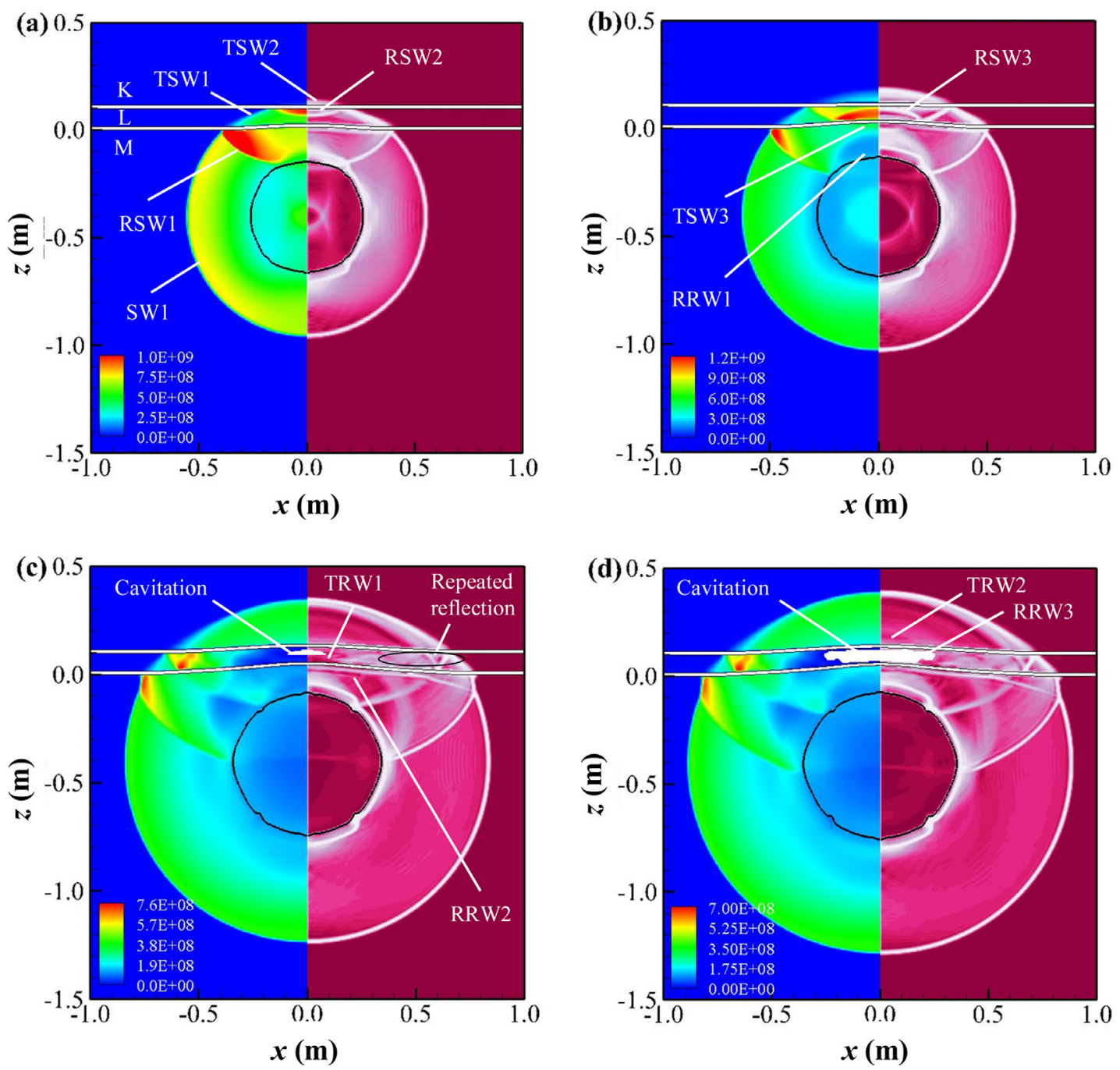

Fig. 19. Pressure distributions (left) and schlieren images (right) for double plates corresponding to $t \approx 0.148$ (a), 0.180 (b), 0.280 (c) and 0.304 (d) ms.

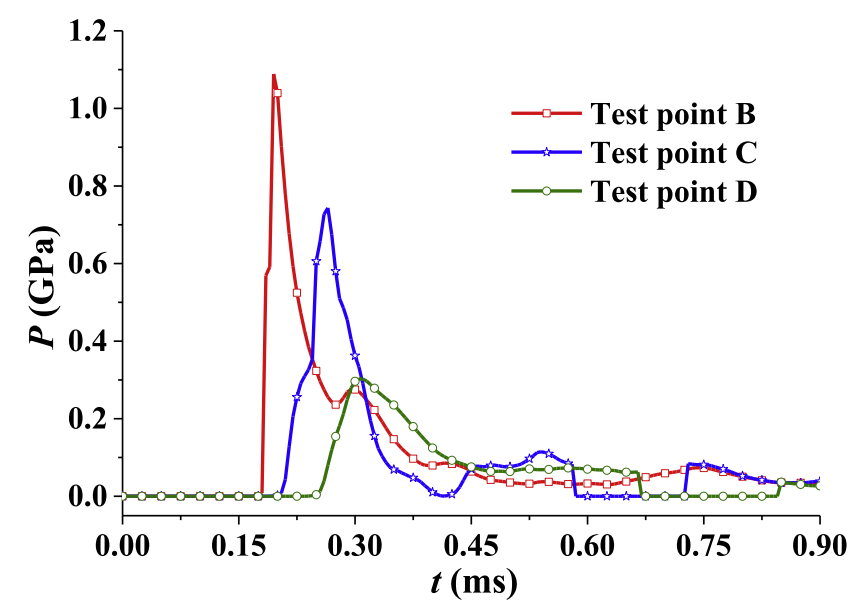

Fig. 20. Evolution of pressure at points B, C and D (see Fig. 18).

The distribution of equivalent plastic strain is shown in Fig. 23. As SW1 arrived at Plate I, the initial plastic deformation of this plate was caused (Fig. 23(a)). After that, deformation of Plate I increased gradually. Plate II was subsequently impacted by TSW1 and RSW3, resulting in a plastic deformation (Fig. 23(b)). With the propagation of multiple waves, Plates I and II continued to deform (Fig. 23(c)). Apparently, the plastic deformation of Plate I was larger than that of Plate

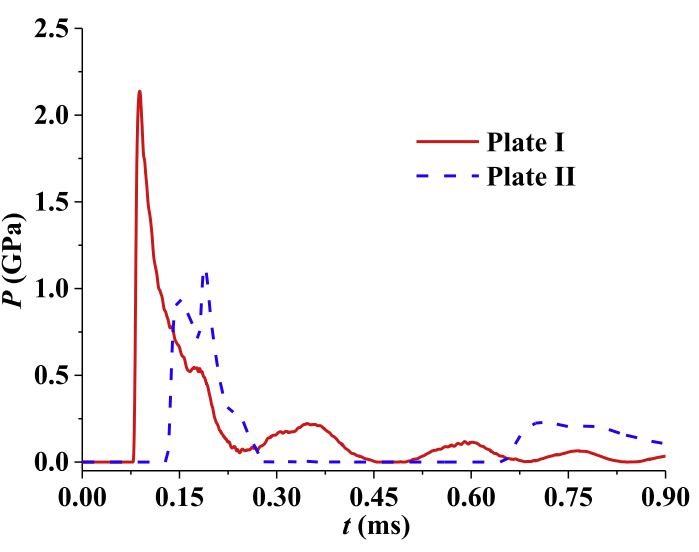

Fig. 21. Evolution of pressure at centre of Plates I and II.

II.

A further comparison of evolutions of displacement and velocity at the centre of Plates I and II is shown in Fig. 24. Plates I and II moved upwards under the effect of SW1 and TSW1, respectively. The levels of displacement and velocity peak of Plate I were larger than those of Plate II because pressure of SW1 was higher than that of TSW1. The velocity of Plate II was close to that of Plate I at the later stage.

Evolution of acceleration at the centre of Plates I and II is illustrated 

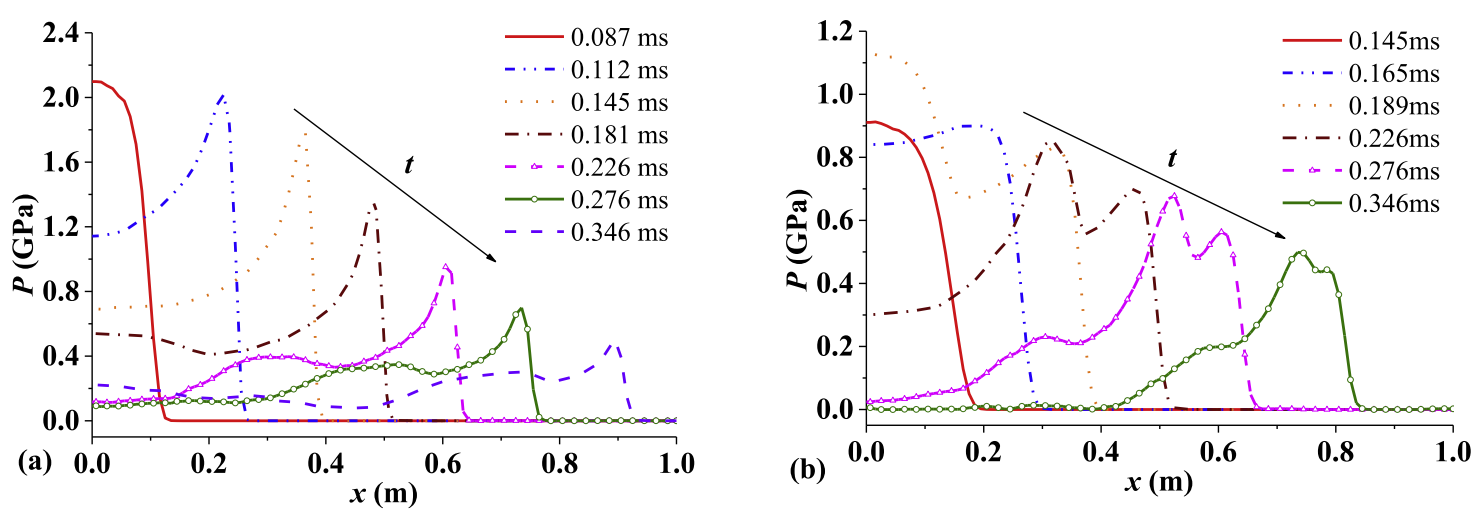

Fig. 22. Pressure distribution at Plates I (a) and II (b).

(a) PEEQ 7 (平均: $75 \%$ )

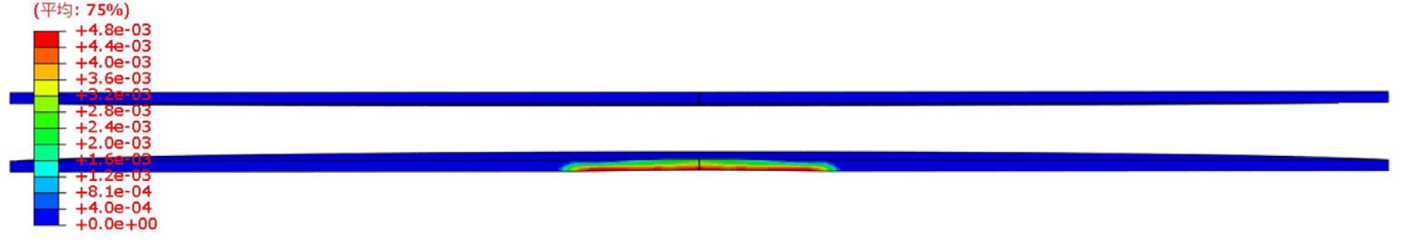

(b) PEEQ 7 (平均: $75 \%)$

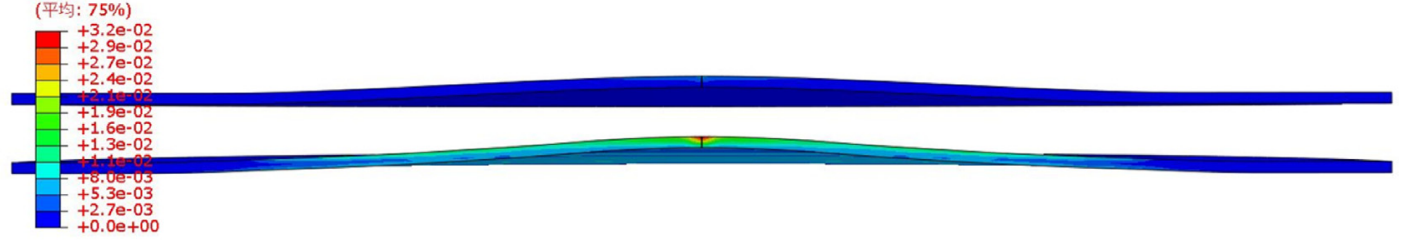

(c) PEEQ

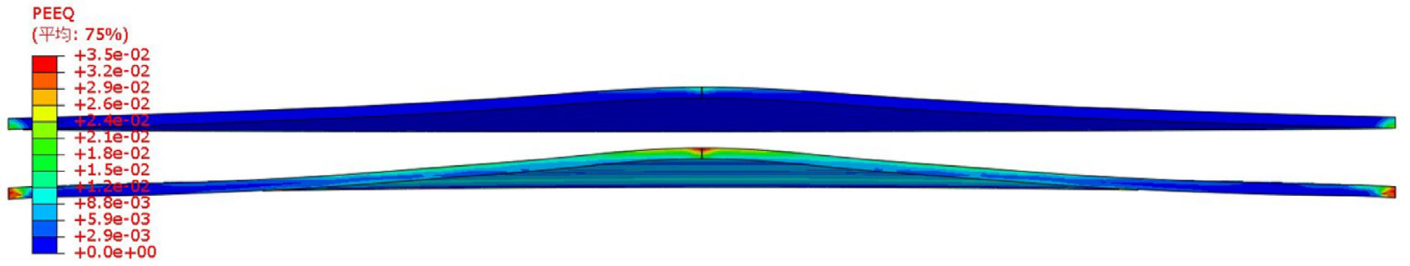

Fig. 23. Distribution of equivalent plastic strain in double plates corresponding to $t \approx 0.102$ (a), 0.300 (b) and 0.448 (c) ms.

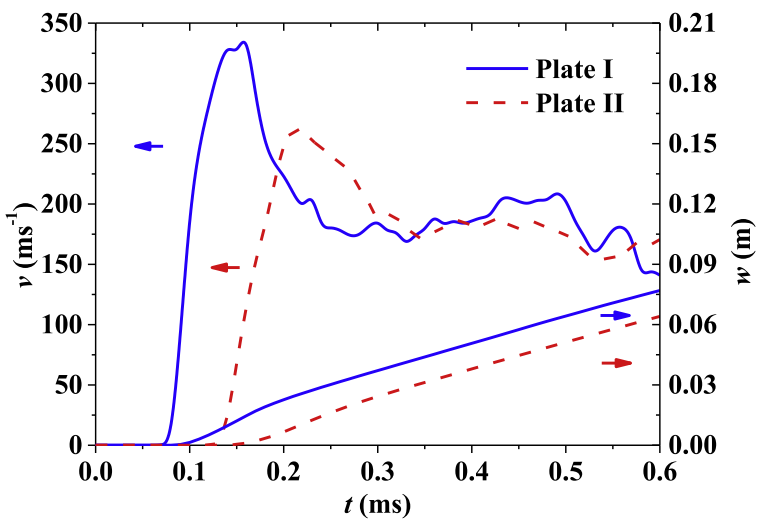

Fig. 24. Evolutions of velocity and displacement of Plates I and II.

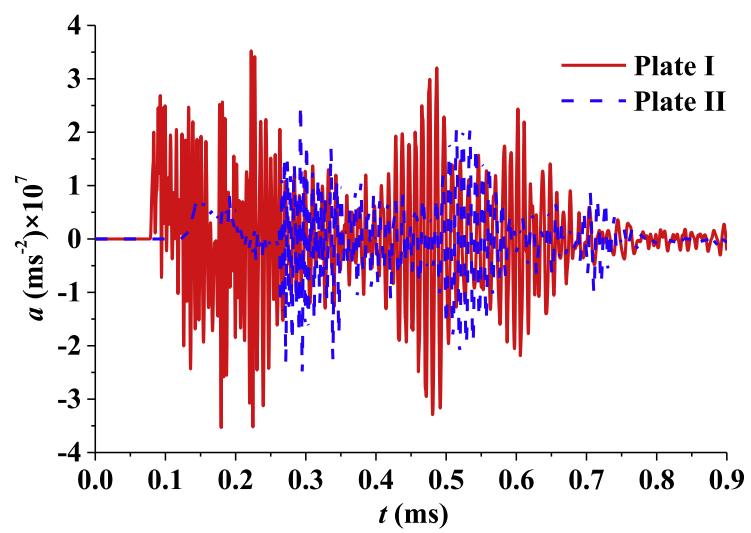

Fig. 25. Evolution of acceleration of Plates I and II. in Fig. 25. The vibration of Plate I impacted by SW1 was more significant than that of Plate II by TSW1. Besides, the shock wave reached Plate I earlier, causing its vibration before that of Plate II. With the decline of the shock load, the amplitude of vibration gradually diminished, with repeated vibration close to the equilibrium position eventually.

3.3.4. Effects of distance on load characteristics

The distance between two plates has a significant effect on load characteristics. Hence, the effect of this parameter is discussed in this 

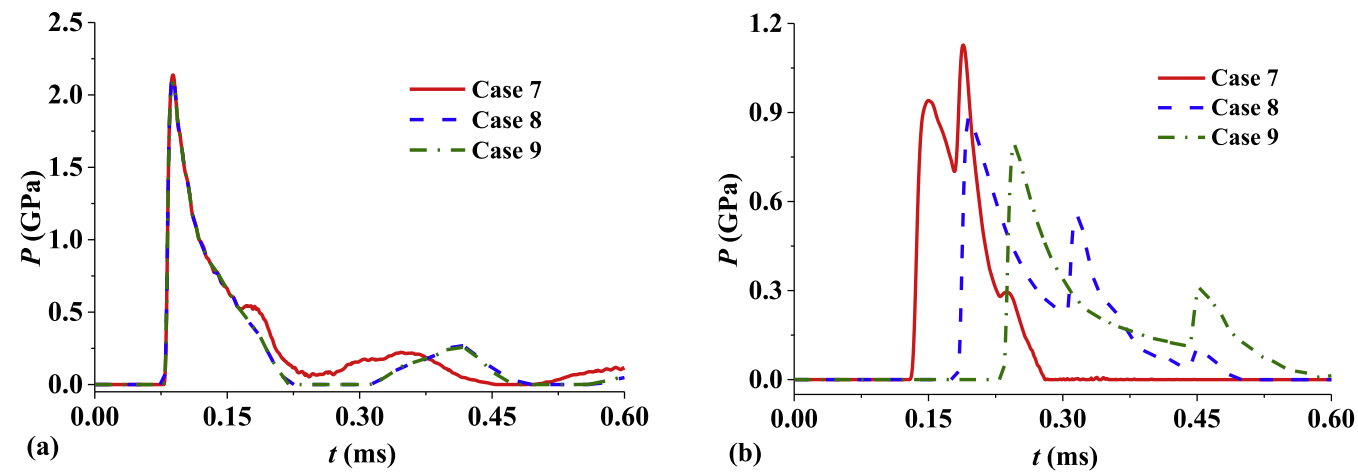

Fig. 26. Evolution of pressure at centre of Plates I (a) and II (b).
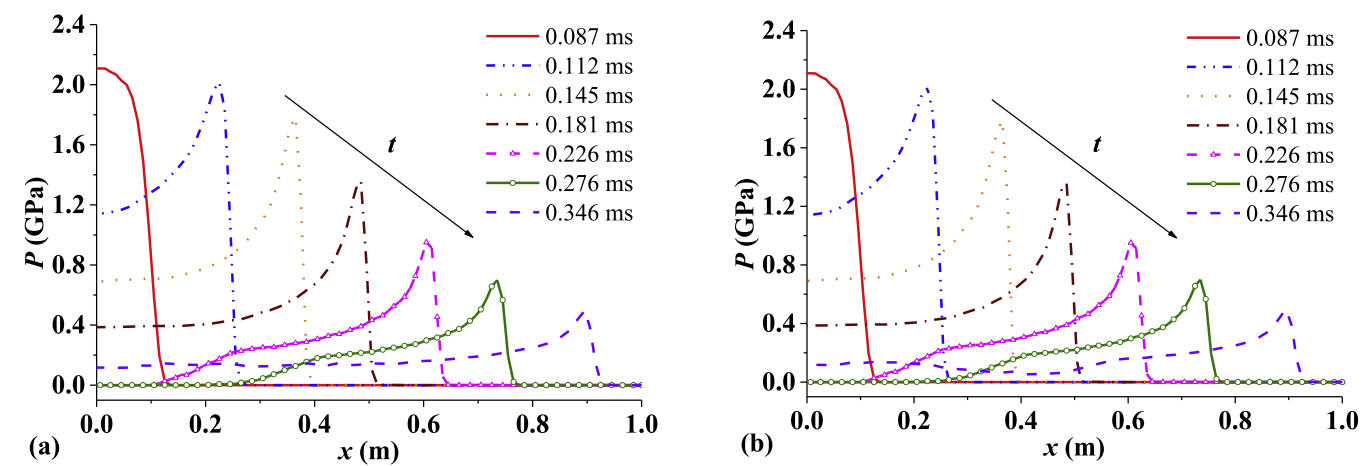

Fig. 27. Pressure distributions at centre of Plate I in Cases 8 (a) and 9 (b).
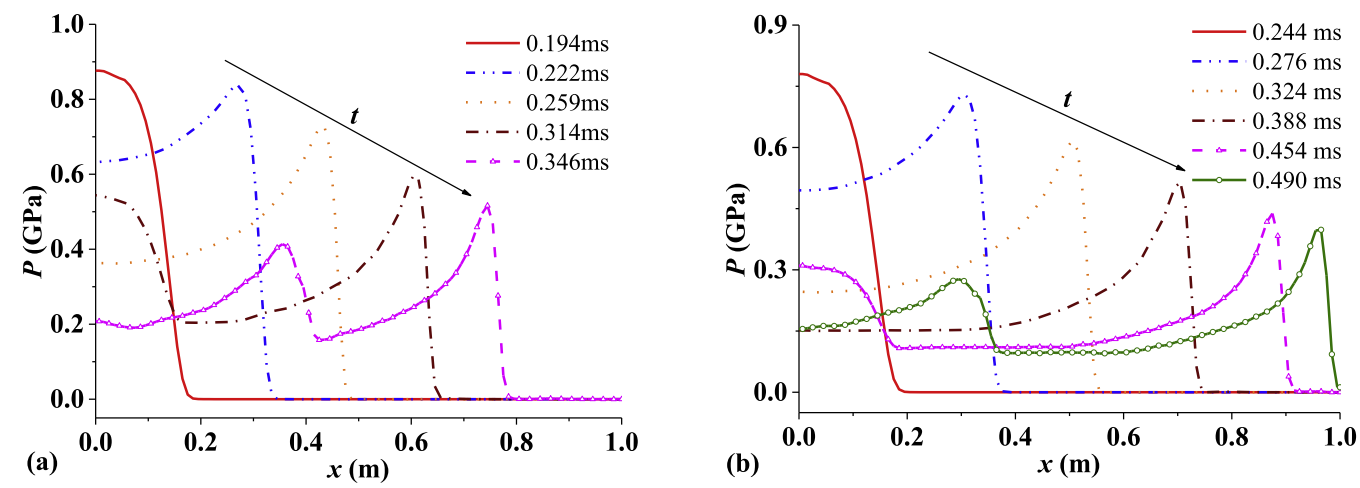

Fig. 28. Pressure distributions at the centre of Plate II in Cases 8 (a) and 9 (b).
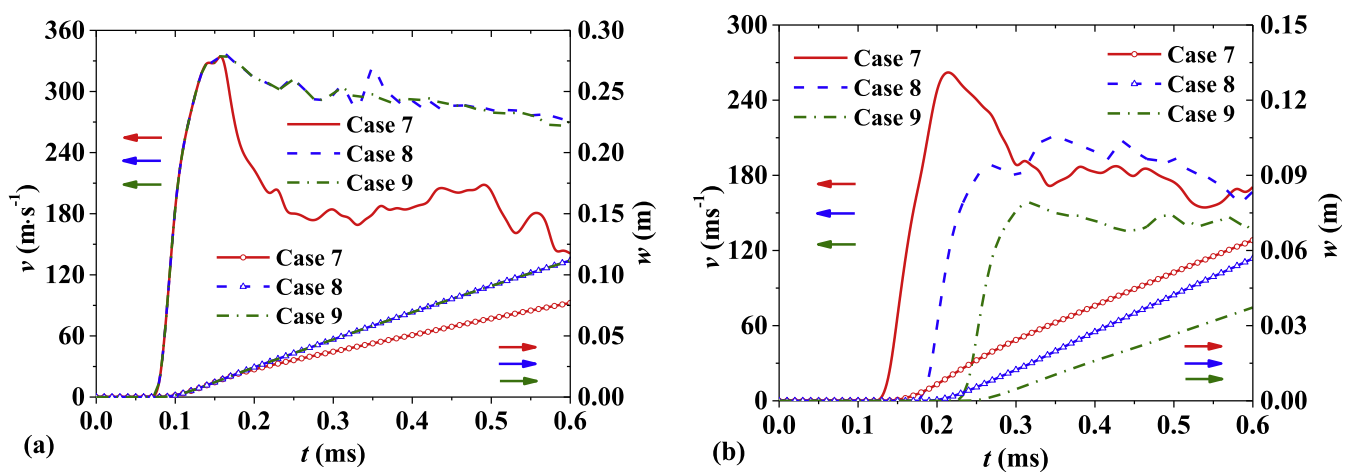

Fig. 29. Evolutions of velocity and displacement at centre of Plates I (a) and II (b).

section, with distances of $0.1 \mathrm{~m}$ (Case 7), $0.2 \mathrm{~m}$ (Case 8) and $0.3 \mathrm{~m}$ (Case 9) studied.

Evolutions of pressure at the centres of Plates I and II are shown in Figs. 26, respectively. The initial tendency and pressure-peak values at the centre of Plate I were similar for the three cases (Fig. 26(a)). The cavitation was formed due to the superposition of RRW1 and RRW2 reflected, respectively, from the bubble and the plate in Cases 8 and 9 while the level of pressure of TSW1 and TRW1 was so high that no 
cavitation was formed in Case 7.

As for Plate II, two peaks were caused by TSW1 and RSW3 (Fig. 26(b)), respectively. The level of the second peak was higher than the first one in Case 7, but not in Cases 8 and 9. With the decrease in distance, these two waves arrived at Plate II earlier and the levels of the two peaks were higher. Besides, the cavitation was formed in a shorter time for a lower distance.

A comparison of pressure distributions at the centre of Plate I in Cases 8 and 9 is presented in Fig. 27. It can be found from the presented comparison results that the distributions for these two cases were similar. After the shock wave reflected from Plate II arrived at Plate I, the intensity of the generated reflected and the transmission shock waves were weaker, resulting in a low effect on the pressure level at Plate I.

The pressure distributions at the centre of Plate II in Cases 8 and 9 are illustrated in Fig. 28. The levels of pressure at Plate II in Case 8 were higher and decreased faster than that in Case 9. With the decline of the distance between the plates, the shock wave reflected by Plate I reached Plate II earlier. The farther from the centre, the lower were the pressure magnitude and the impact force on the plate.

Evolutions of velocity and displacement at the centre of Plates I and II are shown in Fig. 29. The velocity diminished rapidly in Case 7 when RSW2 arrived at Plate I (Fig. 29(a)), with lower deflection than in the other two cases. Due to the larger distance between two plates in Cases 8 and 9, the effect of the reflected shock wave was lower, resulting in a similar tendency for the velocity and displacement. As for Plate II, the smaller the distance parameter, the earlier the arrival of TSW1 at Plate II (Fig. 29(b)). With the decrease of the distance, the level of velocity and deflection were larger.

\section{Conclusions}

A coupled algorithm, fully utilizing advantages of both RKDG and FEM, is applied to capture a strongly discontinuous shock wave and to analyse a shock response of single and double plates subjected to a nearfield underwater explosion of cylindrical charge. The LSM and GFM were used for the update of coordinates of structures and treatment of fluid-structure interaction, respectively. Besides, a cavitation model was introduced into the RKDG method to investigate formation and collapse of the cavitation as well as its secondary shock. First, the RKDG-FEM model of a plate subjected to a near-field underwater explosion of a spherical charge was developed; its results were compared with experimental data to verify the validity of the developed algorithm. Then, the pressure characteristics and the cavitation effect as well as the shock response of single and double plates subjected to the near-field underwater explosion of a cylindrical charge were analysed. Besides, effects of different parameters were discussed. The following main conclusion can be drawn:

(1) A shock wave was generated and propagated in the fluid after the cylindrical charge was detonated. When this shock wave arrived at the plate, a reflected shock wave and a transmitted shock wave were produced in the water. After the reflected shock wave reached the bubble with lower impedance, a rarefaction wave was generated and subsequently reflected, resulting in generation of another rarefaction wave. Due to the superposition of these two rarefaction waves, a cavitation with low pressure was formed. This cavitation subsequently collapsed, causing a secondary shock on structures, and it will cause serious damage to structures. This is consistent with the underwater explosion phenomenon of spherical charge $[29,31]$.

(2) With the decline of the distance between the single plate and the charge, the pressure peak, deformation and velocity as well as acceleration at the centre of the plate increased. With increased plate thickness, the intensity of the transmission shock wave was lower, resulting in a higher pressure peak at the centre of the plate. Besides, reloading caused by the collapse of the cavitation was higher while the magnitudes of deformation and velocity were lower.

(3) After the cylindrical charge exploded underwater near the plates, as for double plates, multiple waves were reflected repeatedly in water between the plates, leading to a more complex pressure distribution than that for the single plate. When the distance between two plates was lower in case 7 , the cavitation process was first formed in the inter-plate area while it occurred near the bottom surface of Plate I at first and, subsequently, in water between the plates when the distance was larger in Case 9. In some cases, multiple cavitation zones were caused. The cavitation zone changes violently with time.

(4) The distance between two plates had a greater effect on the shock response of Plate II. With the increase of this distance, the levels of two pressure peaks caused, respectively, by the transmission shock wave (TSW1) and the reflected shock wave (RSW3) diminished. Besides, the deflection and the maximum velocity were lower.

\section{Acknowledgment}

This work was supported by China Postdoctoral Science Foundation (Grant no. 2017M620644), the National Natural Science Foundation of China (Grant nos. 11732003 and 11521062) and Science Challenge Project (Grant no. TZ2016001). The authors would like to express their sincere thanks to Xi Ye and Liang Ge for helpful comments.

\section{References}

[1] Schiffer A, Tagarielli VL. One-dimensional response of sandwich plates to underwater blast: fluid-structure interaction experiments and simulations. Int J Impact Eng 2014;71:34-49.

[2] Perotti LE, Deiterding R, Inaba K, Shepherd J, Ortiz M. Elastic response of waterfilled fiber composite tubes under shock wave loading. Int J Solids Struct 2013;50(3-4):473-86

[3] Hung CF, Hsu PY, Hwang-Fuu JJ. Elastic shock response of an air-backed plate to underwater explosion. Int J Impact Eng 2005;31(2):151-68.

[4] Ming FR, Zhang AM, Xue YZ, Wang SP. Damage characteristics of ship structures subjected to shockwaves of underwater contact explosions. Ocean Eng 2016;117:359-82.

[5] Zhang ZF, Wang LK, Silberschmidt VV. Damage response of steel plate to underwater explosion: effect of shaped charge liner. Int J Impact Eng 2017;103:38-49.

[6] Cole RH. Underwater explosion. New Jersey: Princeton University Press; 1948. p. $118-27$.

[7] Sternberg HM. Underwater detonation of pentolite cylinders. Phys Fluids 1987;30(3):761-9.

[8] Hammond L. Underwater shock wave characteristics of cylindrical charges. Def Sci Technol Organ 1995. AMRL, DSTO-GD-0029.

[9] Huang C, Wang B, Liu CL, Wang YP, Wang DW. On the shape evolution of underwater explosion bubbles by cylindrical charges. Chin J High Press Phys 2011;25(3):235-41.

[10] Zhang AM, Yang WS, Huang C, Ming FR. Numerical simulation of column charge underwater explosion based on SPH and BEM combination. Comput Fluids 2013;71(3):169-78.

[11] Wang GH, Zhang SR, Yu M, Li HB, Kong Y. Investigation of the shock wave propagation characteristics and cavitation effects of underwater explosion near boundaries. Appl Ocean Res 2014;46(2):40-53.

[12] Makinen K. Cavitation models for structures excited by a plane shock wave. J Fluids Struct 1998;12(1):85-101.

[13] Azarboni HR, Darvizeh A, Darvizeh M, Ansari R. Analysis of cavitation time effect on elastoplastic response of underwater rectangular plate subjected to impulsive loading. Meccanica 2016:52(1-2):1-16.

[14] Gong SW, Lam KY. On attenuation of floating structure response to underwater shock. Int J Impact Eng 2006;32(11):1857-77.

[15] Daramizadeh A, Ansari MR. Numerical simulation of underwater explosion near airwater free surface using a five-equation reduced model. Ocean Eng 2015;110:25-35.

[16] Taylor GI. The pressure and impulse of submarine explosion waves on plates. Compend Underw Explos Res 1950;1:1155-74.

[17] Bleich HH, Sandler IS. Interaction between structures and bilinear fluids. Int J Solids Struct 1970;6:617-39.

[18] Li HT, Zhu X, Huang XM, Mu JL. On the characteristics of cavitation formation subjected to underwater blast shock wave. Chin J High Press Phys 2008;22(2):181-6.

[19] Brett JM, Yiannakopoulos G, Schaaf PJVD. Time-resolved measurement of the deformation of submerged cylinders subjected to loading from a nearby explosion. Int J Impact Eng 2000;24(9):875-90.

[20] Rajendran R. Reloading effects on plane plates subjected to non-contact underwater 
explosion. J Mater Process Technol 2008;206:275-81.

[21] Felippa CA, DeRuntz JA. Finite element analysis of shock-induced hull cavitation. Comput Methods Appl Mech Eng 1984;44(3):297-337.

[22] Wardlaw AB, Luton JA. Fluid-structure interaction mechanisms for close-in explosions. Shock Vib 2000;7:265-75.

[23] Sprague MA, Geers TL. A spectral-element/finite-element analysis of a ship-like structure subjected to an underwater explosion. Comput Methods Appl Mech Eng 2006;195(17-18):2149-67.

[24] Liu TG, Khoo BC, Xie WF. Isentropic one-fluid modelling of unsteady cavitating flow. J Comput Phys 2004;201(1):80-108.

[25] Xie WF, Liu TG, Khoo BC. Application of a one-fluid model for large scale homogeneous unsteady cavitation: the modified Schmidt model. Comput Fluids 2006;35(10):1177-92.

[26] Xie WF, Liu TG, Khoo BC. The simulation of cavitating flows induced by underwater shock and free surface interaction. Appl Numer Math 2007;57(5):734-45.

[27] Zhang AM, Ren SF, Li Q, Li J. 3D numerical simulation on fluid-structure interaction of structure subjected to underwater explosion with cavitation. Appl Math Mech 2012;33(9):1191-206.

[28] Goncalvès E, Charrière B. Modelling for isothermal cavitation with a four-equation model. Int J Multiph Flow 2014;59:54-72.

[29] Park J. A coupled Runge Kutta Discontinuous Galerkin-Direct Ghost Fluid (RKDGDGF) method to near-field early-time underwater explosion (UNDEX) simulations PhD. thesis Virginia: Polytechnic Institute and State University; 2008. p. 1-130.

[30] Wang LK, Zhang ZF, Wang SP. Pressure characteristics of bubble collapse near a rigid wall in compressible fluid. Appl Ocean Res 2016;59:183-92.

[31] Ye X. Study on the noise and near-field load characteristics of bubble under water PhD. thesis Harbin Engineering University; 2014. (In Chinese)

[32] Qiu J, Liu T, Khoo BC. Runge-Kutta discontinuous Galerkin methods for compressible two-medium flow simulations: one-dimensional case. J Comput Phys 2007;222(1):353-73.

[33] Martinelli SL. An Application of the level set method to underwater acoustic propagation. Commun Comput Phys 2012;12(5):1359-91.
[34] Daniel H, Matthias M, Wolfgang S. The constrained reinitialization equation for level set methods. J Comput Phys 2010;229(5):1514-35.

[35] Liu TG, Khoo BC, Yeo KS. The simulation of compressible multi-medium flow: II Application to 2D underwater shock refraction. Comput Fluids 2001;30:315-37.

[36] Liu TG, Khoo BC, Yeo KS, Wang C. Underwater shock-free surface-structure interaction. Int J Numer Methods Eng 2003;58(4):609-30.

[37] Osher S, Fedkiw R. Level set methods and dynamic implicit surfaces. New York: Springer Verlag; 2003.

[38] Fedkiw RP, Marquina A, Merriman B. An isobaric fix for the overheating problem in multimaterial compressible flows. J Comput Phys 1999;148(2):545-78.

[39] Cowper GR, Symonds PS. Strain hardening and strain rate effect in the impact loading of cantilever beams. Small Bus Econ 1957;31(3):235-63.

[40] Peng Y, Deng WY, Xu P, Yao SG. Study on the collision performance of a composite energy-absorbing structure for subway vehicles. Thin Walled Struct 2015;94:663-72.

[41] Cockburn B, Shu CW. TVB Runge-Kutta local projection discontinuous Galerkin finite element method for conservation laws II: General framework. Math Comput 1989;52(186):411-35.

[42] Cockburn B, Lin SY, Shu CW. TVB Runge-Kutta local projection discontinuous Galerkin finite element method for conservation laws III: one-dimensional systems. J Comput Phys 1989;84(1):90-113.

[43] Cockburn B, Hou S, Shu CW. The Runge-Kutta local projection discontinuous Galerkin finite element method for conservation laws IV: the multidimensional case. Math Comput 1990;54:545-81.

[44] Qiu J, Liu TG, Khoo BC. Simulations of compressible two-medium flow by Runge-Kutta discontinuous Galerkin methods with the ghost fluid method. Commun Comput Phys 2008;3(2):479-504.

[45] Dassault Systèmes Simulia Corp. ABAQUS user's and theory manuals, version 6.8., RI, USA: Dassault Systèmes; 2008.

[46] Jacob N, Nurick GN, Langdon GS. The effect of stand-off distance on the failure of fully clamped circular mild steel plates subjected to blast loads. Eng Struct 2007;29(10):2723-36. 